\title{
Imaging of breast implants-a pictorial review
}

\author{
Sergi Juanpere • Elsa Perez • Oscar Huc • \\ Naiara Motos • Josep Pont • Salvador Pedraza
}

Received: 11 March 2011 /Revised: 23 June 2011 / Accepted: 18 July 2011 /Published online: 7 August 2011

(C) European Society of Radiology 2011

\begin{abstract}
The number of women with breast implants is increasing. Radiologists must be familiar with the normal and abnormal findings of common implants. Implant rupture is a well-known complication after surgery and is the main cause of implant removal. Although mammography and ultrasonography are the standard first steps in the diagnostic workup, magnetic resonance imaging (MRI) is the most useful imaging modality for the characterisation of breast implants because of its high spatial resolution and contrast between implants and soft tissues and absence of ionising radiation. MRI has the highest sensitivity and specificity for implant rupture, thanks to its sequences that can suppress or emphasise the signal from silicone. Regardless of the technique used, the overall aim of imaging breast implants is to provide essential information about tissue and prosthesis integrity, detect implant abnormalities and detect breast diseases unrelated to implants, such as breast cancer.
\end{abstract}

\footnotetext{
S. Juanpere $\cdot$ E. Perez $\cdot$ N. Motos $\cdot$ J. Pont $\cdot$ S. Pedraza

Department of Radiology-IDI,

Dr. Josep Trueta University Hospital,

Girona, Spain

O. Huc

Department of Plastic Surgery,

Dr. Josep Trueta University Hospital,

Girona, Spain

S. Juanpere $(\bowtie)$

Department of Diagnostic Radiology,

Dr. Josep Trueta University Hospital,

Av Francia s/n,

Girona, Spain

e-mail: sejuanp@hotmail.com
}

Keywords Breast implants · Implant rupture · Magnetic resonance imaging $\cdot$ Mammography $\cdot$ Ultrasonography

\section{Introduction}

An increasing number of patients have breast implants for cosmetic augmentation of the breast, reconstruction after mastectomy or correction of congenital malformations [1]. Implant rupture is the main cause of implant removal. Implant rupture can have various causes, but most ruptures have no obvious traumatic origin and sometimes occur in asymptomatic patients. Most implant ruptures occur 10 to 15 years after implantation [2]. The incidence of rupture increases with implant age; the average incidence is approximately 2 implant ruptures per 100 implant-years, with an estimated probability of being intact after 5 and 10 years of implantation of $98 \%$ and $83-85 \%$, respectively [3-6].

Clinical diagnosis is difficult, being based solely on nonspecific findings such as palpable nodules, asymmetry or tenderness [7]. Free silicone from ruptured implants has in rare cases spread to distant body regions, giving rise to symptoms. If implant rupture is accompanied by loss of the shape of the breast, the diagnosis of breast implant rupture at physical examination is feasible. However, clinical evaluation may fail to detect breast implant rupture that occurs over time without loss of breast volume and misshapenness. Breast pain on the clinical examination of implants is a strong predictor of rupture, but the absence of pain does not exclude rupture [3]. According to Tark et al. [8], the most common symptom in breast implant rupture is contour deformity (44\%), followed by displacements $(20 \%)$, mass formations (17\%), pain (13\%) and inflammation $(3 \%)$. However, physical examination fails to diagnose implant rupture in more than $50 \%$ of cases. 

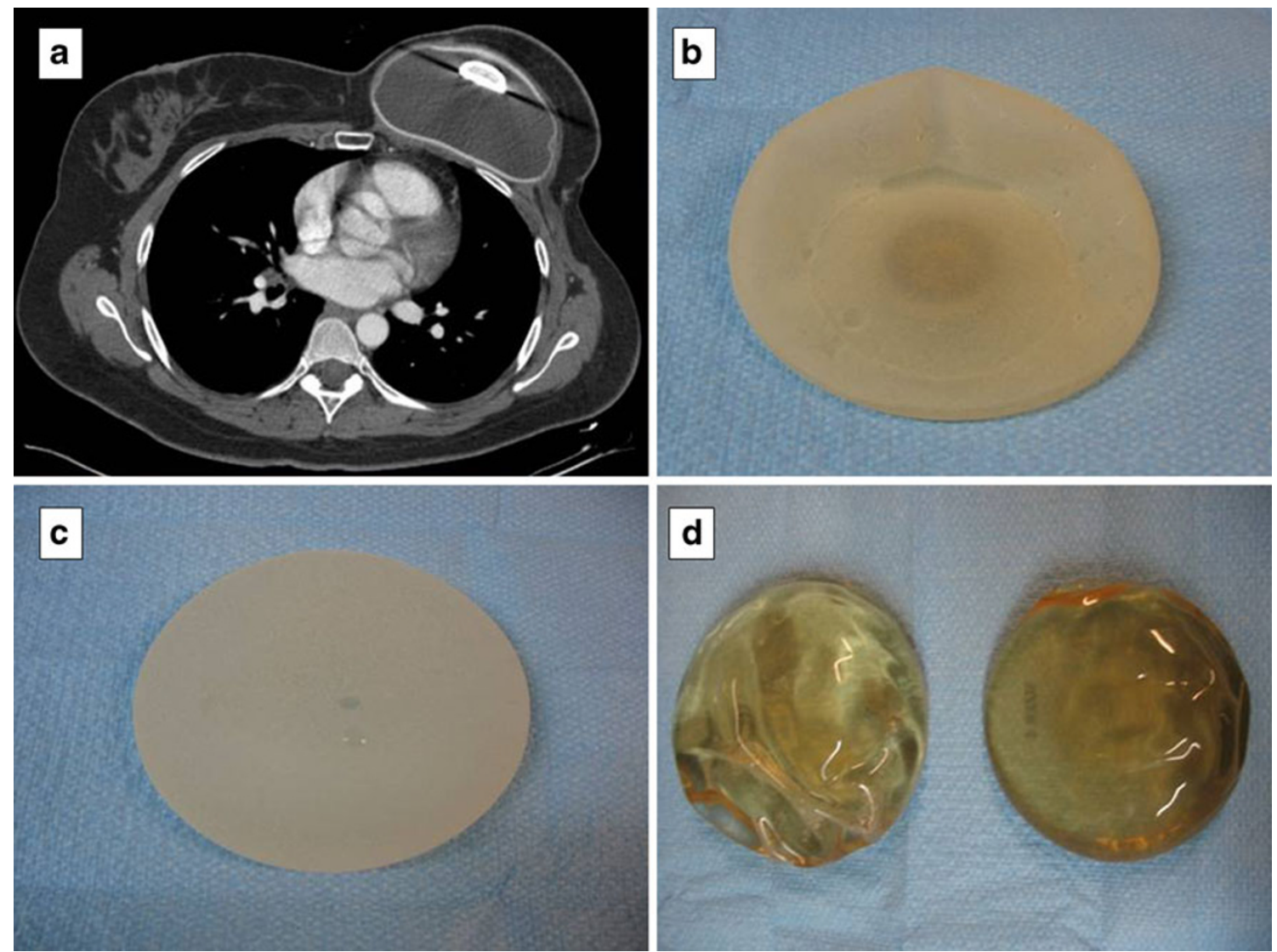

Fig. 1 Different implant types. a and $\mathbf{b}$ Breast tissue expander with metallic component visualised by computed tomography scan. Silicone gel implants with textured surface (c) and with smooth surface (d)

Magnetic resonance imaging (MRI), mammography, ultrasonography (US) and exceptionally computed tomog-

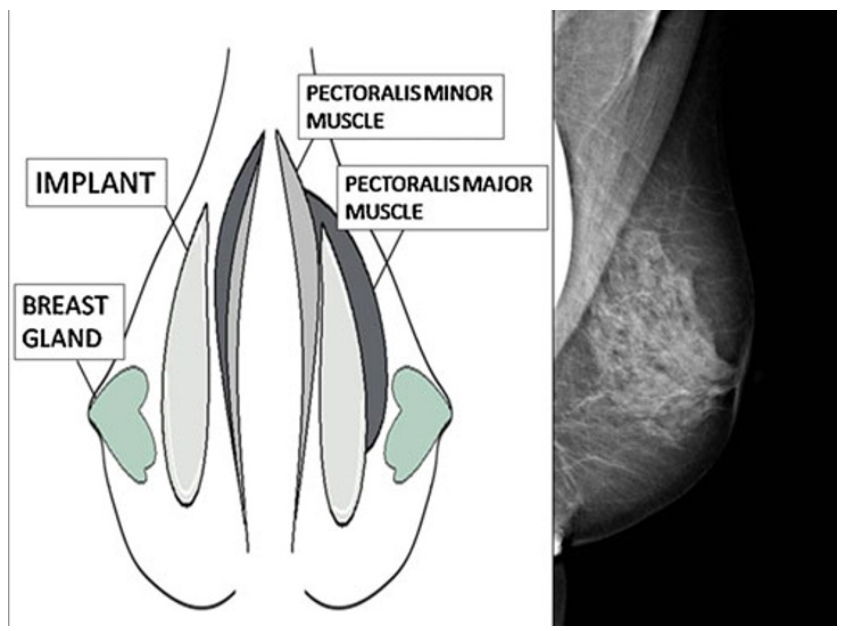

Fig. 2 Localisation of breast implants. (Left) Submammary implant located in front of the pectoralis major muscle and (right) submuscular implant located behind the pectoralis major muscle, visualised by mammography raphy (CT) have all been used to diagnose silicone breast implant rupture. Each technique has specific strengths and weaknesses that may make a particular technique the study of choice for an individual patient [9]. Familiarity with both the typical and atypical findings for implants is essential to

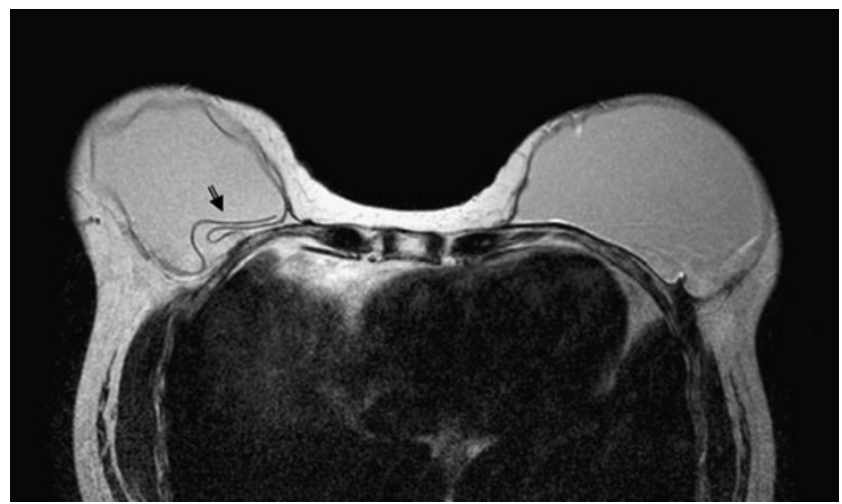

Fig. 3 Magnetic resonance imaging scan of a woman with bilateral breast silicone implants. Right implant with extracapsular rupture exhibiting the typical "linguine sign" at the posterior margin of the implant. Intact left silicone implant 


\begin{tabular}{|c|c|c|c|c|c|c|c|c|}
\hline \multirow{2}{*}{ SEQUENCE } & \multirow{2}{*}{$\begin{array}{l}\text { Th- } \\
\text { weighted } \\
\text { TSE }\end{array}$} & \multirow{2}{*}{$\begin{array}{c}\text { T2- } \\
\text { weighted } \\
\text { TSE }\end{array}$} & \begin{tabular}{|c|} 
DYNAMIC \\
3D
\end{tabular} & $\begin{array}{l}\text { THRNE HR } \\
\text { TI TFR 3D }\end{array}$ & $\begin{array}{l}\text { TI TURBO } \\
\text { SPIN ECHO }\end{array}$ & \multirow{2}{*}{$\begin{array}{l}\text { DIFFUSION- } \\
\text { weighted EPI }\end{array}$} & \multirow{2}{*}{$\begin{array}{c}\begin{array}{c}\text { SILCONE } \\
\text { SUPPRESSION }\end{array} \\
\text { FAT } \\
\text { SUPPRESSION }\end{array}$} & \multirow{2}{*}{\begin{tabular}{|c|}
$\begin{array}{c}\text { SILCONE } \\
\text { EXCITED }\end{array}$ \\
WATER \\
SUPPRESSION
\end{tabular}} \\
\hline & & & \multicolumn{3}{|c|}{ FAT SUPPRESSION } & & & \\
\hline $\begin{array}{l}\text { sequence } \\
\text { time }\end{array}$ & $2,43 \mathrm{~min}$ & $5,19 \mathrm{~min}$ & $6,11 \mathrm{~min}$ & $1,32 \mathrm{~min}$ & $1,08 \mathrm{~min}$ & $1,40 \mathrm{~min}$ & $2,05 \mathrm{~min}$ & $4,09 \mathrm{~min}$ \\
\hline sections & $6 \mathrm{~mm}$ & $4 \mathrm{~mm}$ & - & - & $5 \mathrm{~mm}$ & $5 \mathrm{~mm}$ & - & $3,5 \mathrm{~mm}$ \\
\hline slice & 32 & 45 & 75 & 150 & 17 & 17 & 120 & 38 \\
\hline GAP & 0,6 & 0 & & - & 0,5 & 0,5 & - & 0 \\
\hline matrix & $280 \times 224$ & $524 \times 513$ & $324 \times 237$ & $388 \times 389$ & $232 \times 183$ & $80 \times 35$ & $368 \times 368$ & $272 \times 210$ \\
\hline MSA & 3 & 1 & 1 & 1 & 2 & 2 & 1 & 2 \\
\hline $\begin{array}{c}\text { phase } \\
\text { direction }\end{array}$ & RL & RL & $R L$ & $R L$ & $\mathrm{FH}$ & $\mathrm{FH}$ & $\mathrm{RL}$ & RL \\
\hline $\begin{array}{c}\text { repetition } \\
\text { time }\end{array}$ & $241 \mathrm{~ms}$ & $3800 \mathrm{~ms}$ & $12 \mathrm{~ms}$ & $5,2 \mathrm{~ms}$ & $628 \mathrm{~ms}$ & $2500 \mathrm{~ms}$ & $5,4 \mathrm{~ms}$ & $\begin{array}{c}\text { TR } 5944 \mathrm{~ms} \text { TI } \\
165 \mathrm{~ms} \\
\end{array}$ \\
\hline echo time & $4,6 \mathrm{~ms}$ & $120 \mathrm{~ms}$ & $6,3 \mathrm{~ms}$ & $2,6 \mathrm{~ms}$ & $8 \mathrm{~ms}$ & $68 \mathrm{~ms}$ & $2,7 \mathrm{~ms}$ & $70 \mathrm{~ms}$ \\
\hline
\end{tabular}

Fig. 4 Our MRI examination protocol includes a 1.5-T superconducting MR system (Philips MR Systems Gyroscan NT) with a SENSE-body coil, with the following sequences. We always include a post-contrast study to detect possible malignant lesions

enable abnormalities to be detected. Many factors can influence which imaging technique should be used to evaluate the integrity of silicone breast implants in a particular patient. These factors include the cost of the examination, the availability of an imaging technique, the expertise of the radiologist performing and interpreting the study, and potential contraindications or limitations of a patient that would prevent the use of a specific imaging technique [9].

Furthermore, knowing which implant the patient has can help determine the type of imaging findings to expect in case of rupture. Each type of silicone gel-filled implant has slightly different imaging findings for implant failure related to the manufacturing process and viscosity of the silicone gel.

Breast reconstruction may involve the insertion of various types of implant or the modelling of autologous myocutaneous flaps. According to the literature, breast implants can be categorised into five implant generations reflecting product development over time. The recent generations of silicone gel implants have a cohesive viscous silicone gel. As a result of this feature, these implants will rarely have a totally collapsed implant shell, differing from the older generations. Moreover, most of them have gel leakage and silicone migration. The third and fourth implant generations offered models of breast implants with textured or uniformly smooth surfaces (Fig. 1), and it seems that capsular contracture rarely occurs in women with recent generations of breast implants. All implants in this article are silicone breast implants including single-lumen implants (the majority) and less commonly double-lumen implants (Fig. 5).
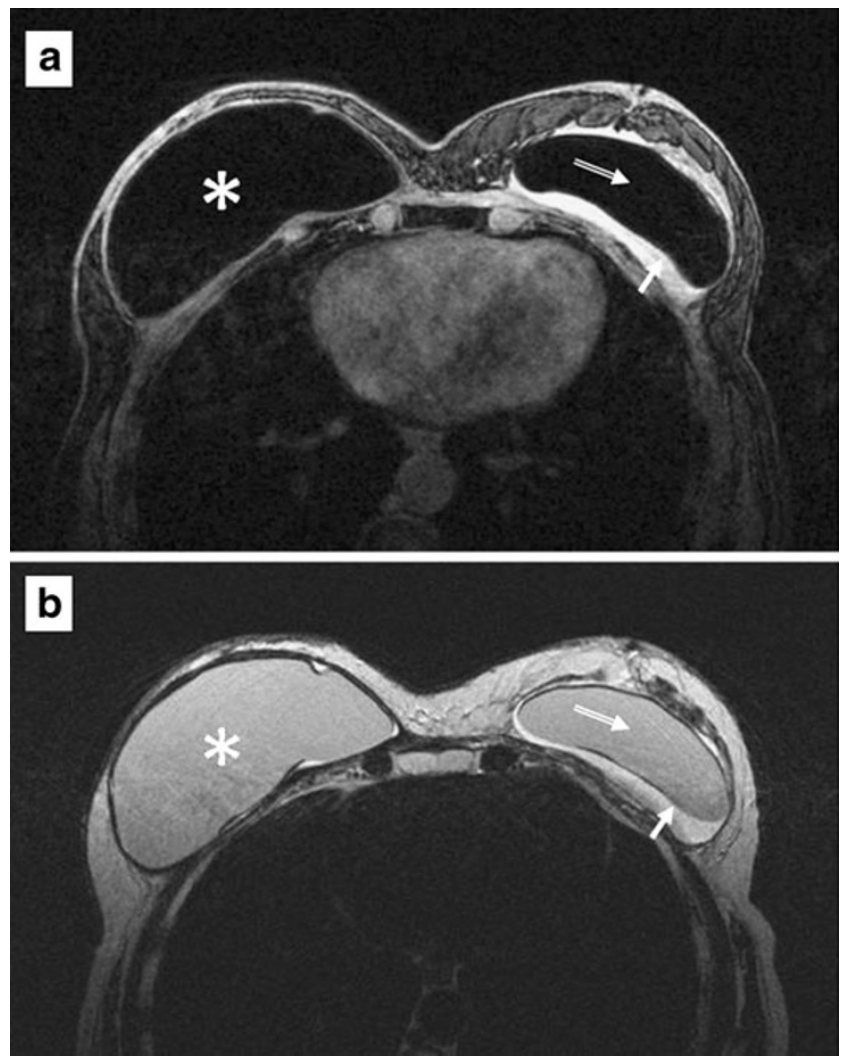

Fig. 5 MRI of a 61-year-old woman with bilateral implants: a single-lumen implant (right breast) and a double-lumen implant (left breast). (a) Axial silicone-suppression and (b) axial T2-weighted turbo spin echo sequences. The right implant has homogeneous signal intensity, representing a single lumen with silicone gel (*). The left implant has an inner lumen (open arrow) of low-signal-intensity or high-signal-intensity silicone surrounded by a smaller outer lumen (solid arrow) that contains saline 


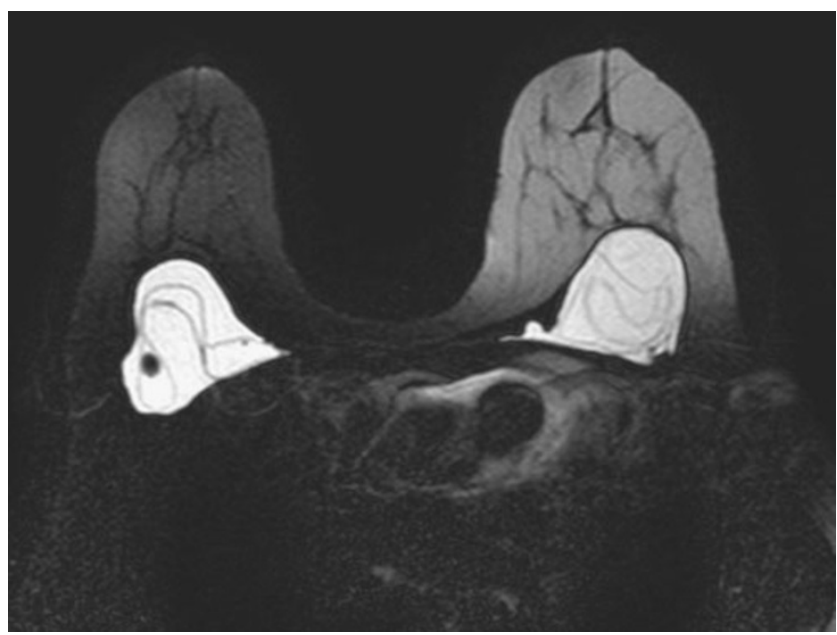

Fig. 6 Magnetic resonance imaging scan of a woman with bilateral ruptured implants. Typical "linguine sign" within implants representing collapsed implant shell

Single-lumen implants have a single lumen of silicone gel delimited by a multilayer shell, while double-lumen implants have a fixed amount of saline and silicone within them, or a fixed outer lumen usually filled with silicone and an inner lumen that can be expanded, as necessary, with saline (inverse double-lumen) [10].

Finally, another surgical breast reconstruction technique uses autologous myocutaneous flaps. Flaps are most commonly either transverse rectus abdominis musculocutaneous (TRAM) flaps or latissimus dorsi flaps, and are used to reform the breast either alone or in conjunction with an implant. Another alternative more rarely encountered in clinical practice is direct silicone gel injection in the breast.
Breast implants may be placed in a subglandular (anterior to the pectoralis major muscle) or subpectoral (posterior to the pectoralis major muscle) location (Fig. 2).

Before implant insertion, especially in oncoplastic breast reconstruction, a tissue expander (Fig. 1) is usually placed in the mastectomy site to stretch the remaining skin in preparation for the placement of a permanent implant later. The expander is like an inflatable breast implant that is inserted into a pocket under the skin and muscle of the chest. The expander is usually placed in its collapsed form at the time of mastectomy and then, after surgery, fluid is introduced into the tissue expander to slowly inflate it. It is important to realise that, as their function is to expand, they might not be fully inflated and thus may appear to have multiple folds or wrinkles on the surface. This should not be interpreted as rupture [10]. Depending on the type of expander, the fluid is either introduced directly into the expander (magnetic marker) or is injected into a distant port. This process continues for several weeks until the tissue expander is filled to an optimal volume, when a permanent breast implant can be inserted.

Notably, some kind of breast tissue expanders should be considered a contraindication to MRI because of the magnetic marker of the filling valve: expander manufacturers list possible consequences such as overheating, possible expander displacement, and possible reduction of magnetisation of the marker [11].

Early postoperative complications of breast augmentation include hematoma and infection [12]. After placement, a thin fibrous capsule (scar tissue) normally forms around the prosthesis. This occurs around all silicone implants to some degree; however, pronounced fibrous capsule forma-

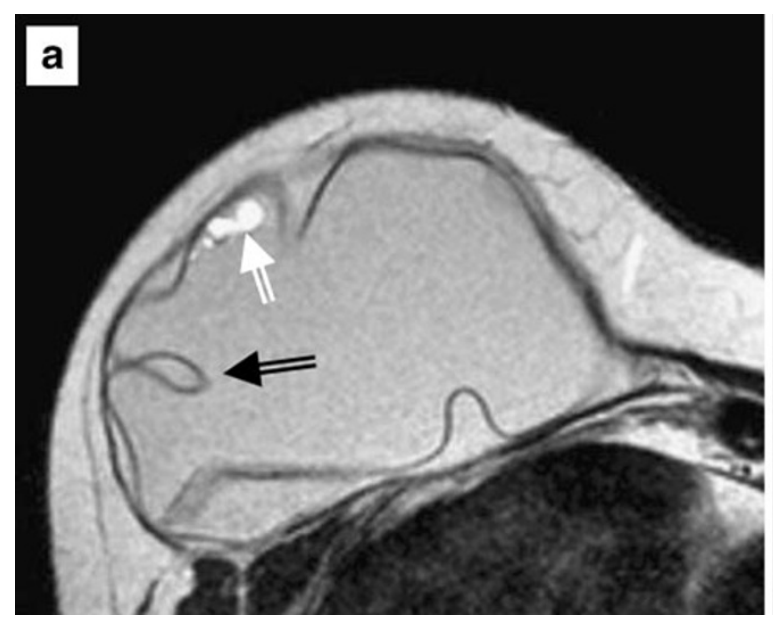

Fig. 7 MRI of a woman with intracapsular rupture of a single-lumen silicone implant. (a) Axial T2-weighted turbo spin-echo and (b) axial silicone-excited sequence. The study shows a hypointense subcapsular line at the anterior margin of the implant (solid arrow); the "teardrop

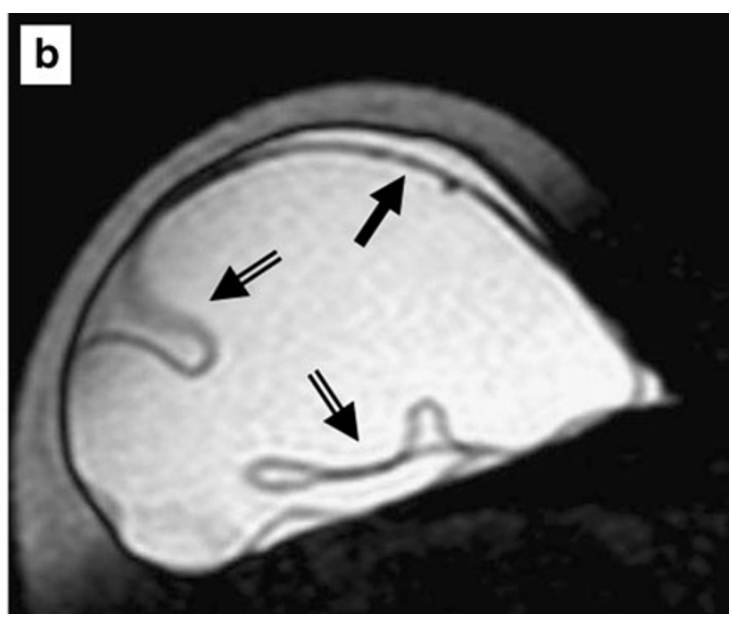

sign" and "key-hole sign" are also present (open arrows). Focal change in signal at the anterior margin of the implant (white open arrow) can also be observed 

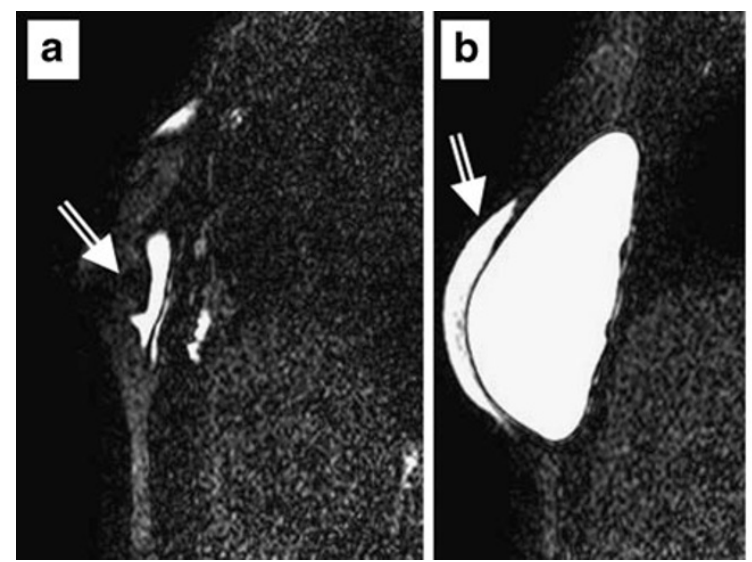

Fig. 8 MRI of a woman with an extracapsular rupture of a singlelumen silicone implant. a and b Sagittal silicone-excited sequences demonstrate the presence of free silicone gel around the implant (white

tion causes discomfort and alters the shape of the breast. This capsular contracture is one of the most common complications of implant insertion. Changes owing to marked fibrous capsule contraction often cannot be appreciated on imaging, and clinical examination is the best way to diagnose this condition [10].

Magnetic resonance imaging (MRI) is the most accurate technique in the evaluation of implant integrity. Its sensitivity for rupture is between $80 \%$ and $90 \%$, and its specificity is between $90 \%$ and $97 \%$ [3, 5-16] (Fig. 3).

MRI may be used to exclude a ruptured prosthesis, and it may aid explantation surgery as it documents the presence and extent of silicone leakage better than other imaging techniques. Despite this, conventional breast imaging techniques are the methods of choice when breast implant failure is suspected because well-defined and discernible sonographic features have been established for ruptured implants [1], as we shall show throughout this article. Moreover, MRI is more expensive, and many women have contraindications (cardiac pacemakers, aneurysm clips, metallic foreign bodies and claustrophobia) to MRI.

In this article, we describe the preeminent role of MRI in detecting implant failures, illustrating the spectrum of appearances of normal silicone gel implants and of implant ruptures. Furthermore, we summarise the advantages and limitations of other imaging techniques and describe the key findings in detection of silicone implant failure.

\section{Magnetic resonance imaging}

Magnetic resonance imaging of silicone breast implants, with its high sensitivity and specificity for detection of

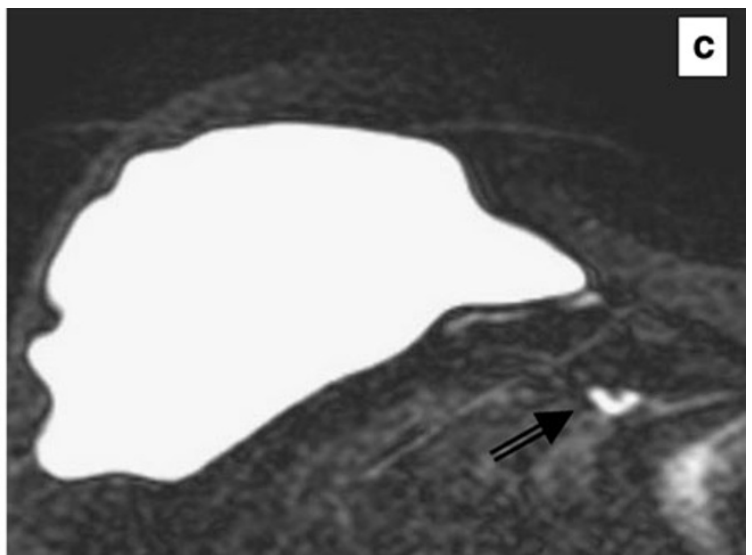

arrows). (c) Axial silicone-excited sequence shows free silicone gel located in the internal mammary chain (black arrow)

rupture, is an excellent technique to assess implant integrity. MRI has proven accurate in locating free silicone and evaluating implant rupture [3,5-16]. A dedicated MRI
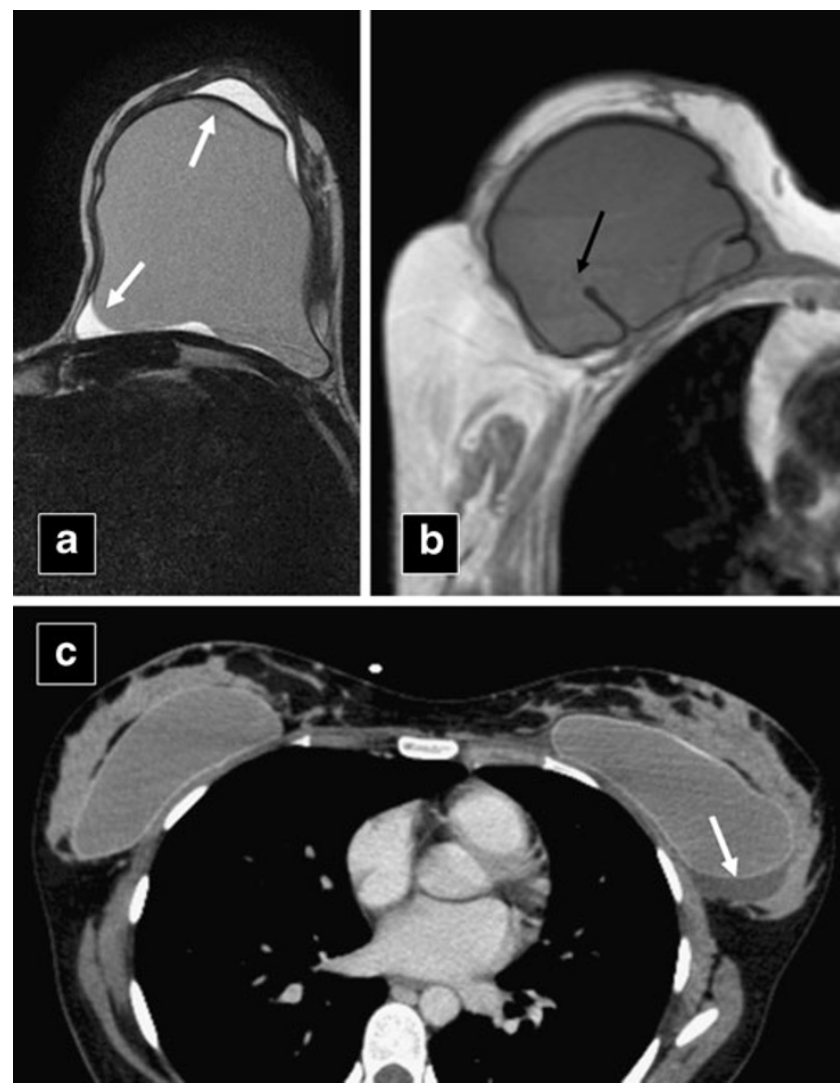

Fig. 9 Axial T2-weighted turbo spin-echo image (a) and axial CT scan (c) of single-lumen implants show small amount of reactive fluid (arrows). (b) Axial T1-weighted turbo spin-echo image demonstrates normal radial folds of the membrane (arrowhead). Simple or complex folds are not in themselves indicative of rupture 


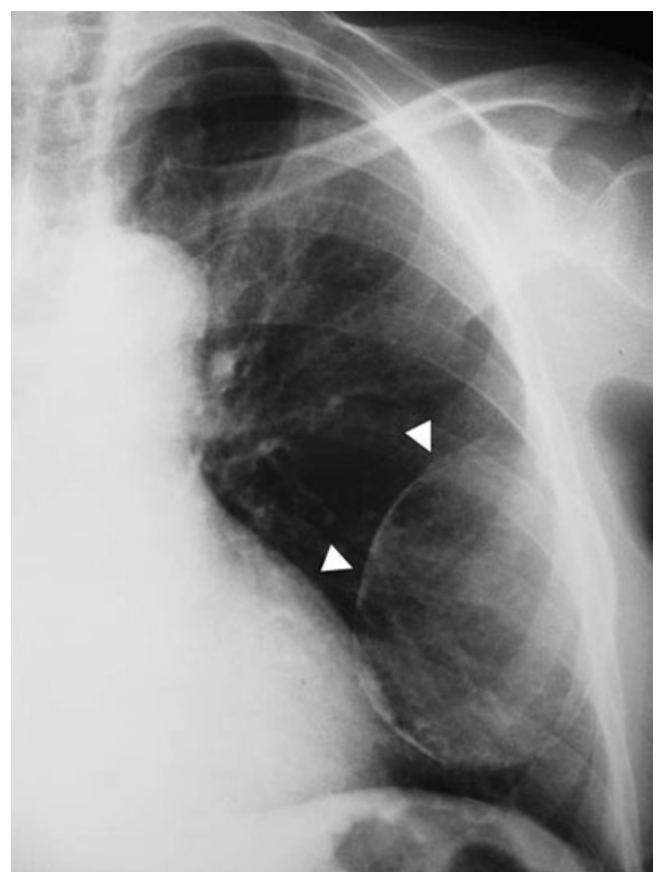

Fig. 10 Chest plain film of a woman with capsule calcification (arrowheads) adjacent to the implant. Many augmented patients develop capsular contracture

breast coil should be used to obtain high-resolution images, allowing detection of subtle signs of implant leakage or rupture. MRI's usefulness derives from its ability to suppress or emphasise the signal from water, fat, or especially silicone. Its high spatial and soft-tissue resolutions make it ideal for the characterisation of breast implants [2].

Multiplanar dedicated T1- and T2-weighted MRI techniques are used for evaluating implant integrity [11]. The lack of ionising radiation is another advantage of MRI.
Figure 4 shows the MRI sequences used at our institution for breast implant study. The use of contrast agents in MRI studies for assessment of breast implant integrity is not recommended. However, when the priority is the detection of recurrence or residual tumors, contrast-enhanced MRI of the breast is useful for characterising parenchymal lesions $[11,13]$. High-field-strength magnets of at least $1.5 \mathrm{~T}$ are preferred when imaging patients with silicone breast implants because of the ability to use magnetic resonance sequences that can more readily suppress or emphasise the signal from water, fat or silicone. Turbospin-echo T2-weighted images, short-time inversion recovery silicone excited (silicone hyperintense, water suppressed), and silicone-saturated (water hyperintense, silicone suppressed) are the most common and most important sequences in silicone breast implant assessment. A single-lumen silicone implant has an outer shell containing homogeneous high-signal-intensity viscous silicone on T2-weighted images. A double-lumen silicone implant typically has an inner lumen of highsignal-intensity silicone surrounded by a smaller outer lumen that contains saline and has different signal intensities, depending on the pulse sequence (Fig. 5).

Breast implant ruptures can be divided into two major categories: intracapsular implant rupture and extracapsular implant rupture, which is less common [1, 3-10, 12, 15-18]. Up to half of all ruptures in women with augmented breasts occur within 12 years [1]. Intracapsular implant rupture is defined as rupture of the implant shell with silicone leakage that does not macroscopically extend beyond the fibrous capsule. The most reliable MRI criterion for intracapsular rupture is the presence of multiple curvilinear low-signalintensity lines within the high-signal-intensity silicone gel,
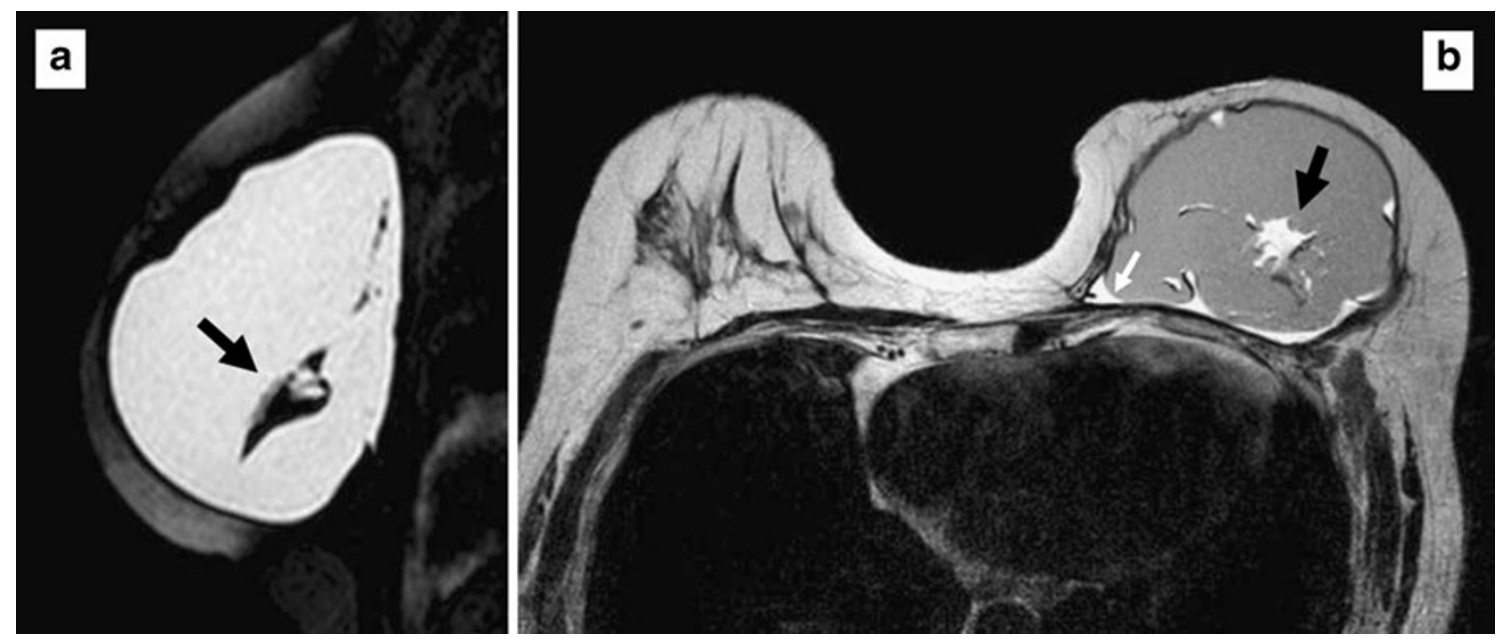

Fig. 11 a Sagittal silicone-excited MRI sequence and (b) axial T2weighted turbo spin-echo image of a 64-year-old woman with changes in the signal intensity of the silicone gel (black arrows). The margins of the implant are slightly irregular and a small amount of fluid surrounds the prosthesis (white arrow). A ruptured implant was confirmed at surgery. 

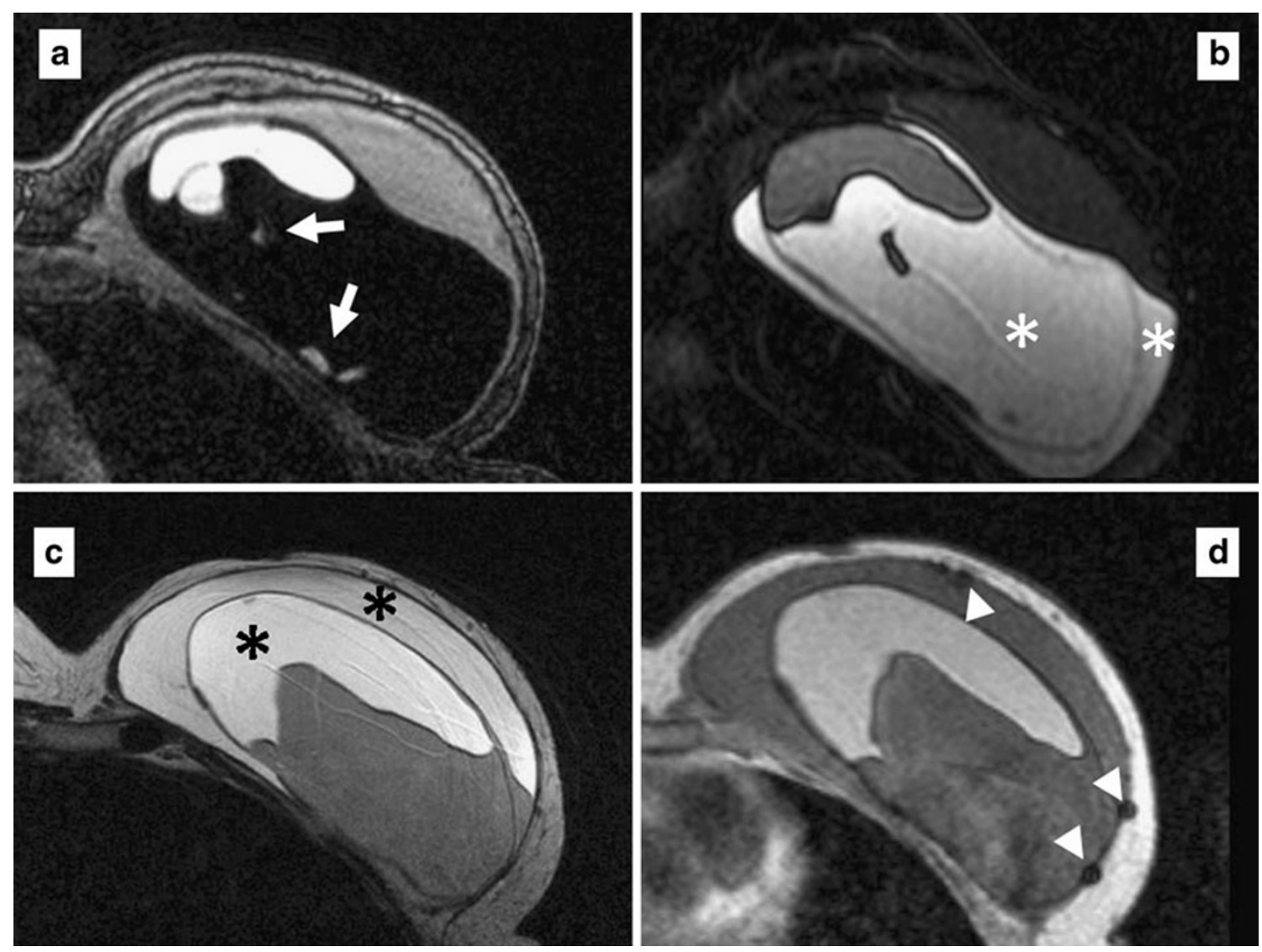

Fig. 12 MRI of a 54-year-old woman with a ruptured breast implant confirmed at surgery. a Axial silicone suppression. b Axial siliconeexcited sequence. c Axial T2-weighted turbo spin echo. d Axial T1weighted turbo spin echo. Silicone gel (white asterisks) inside and outside the implant. A moderate amount of water and probably serum

is mixed in the silicone gel around the implant (black asterisks). Note also the punctuate changes in signal intensity-droplets within the implant (arrows) and punctuate and hyperintense images due to calcifications in the implant periphery (arrowheads)

the so-called "linguine sign". These curvilinear lines represent the collapsed implant shell floating within the silicone

gel $[5,7,9,10,12,13,16]$ (Fig. 6). The linguine sign will not be present in an uncollapsed rupture. In an uncollapsed

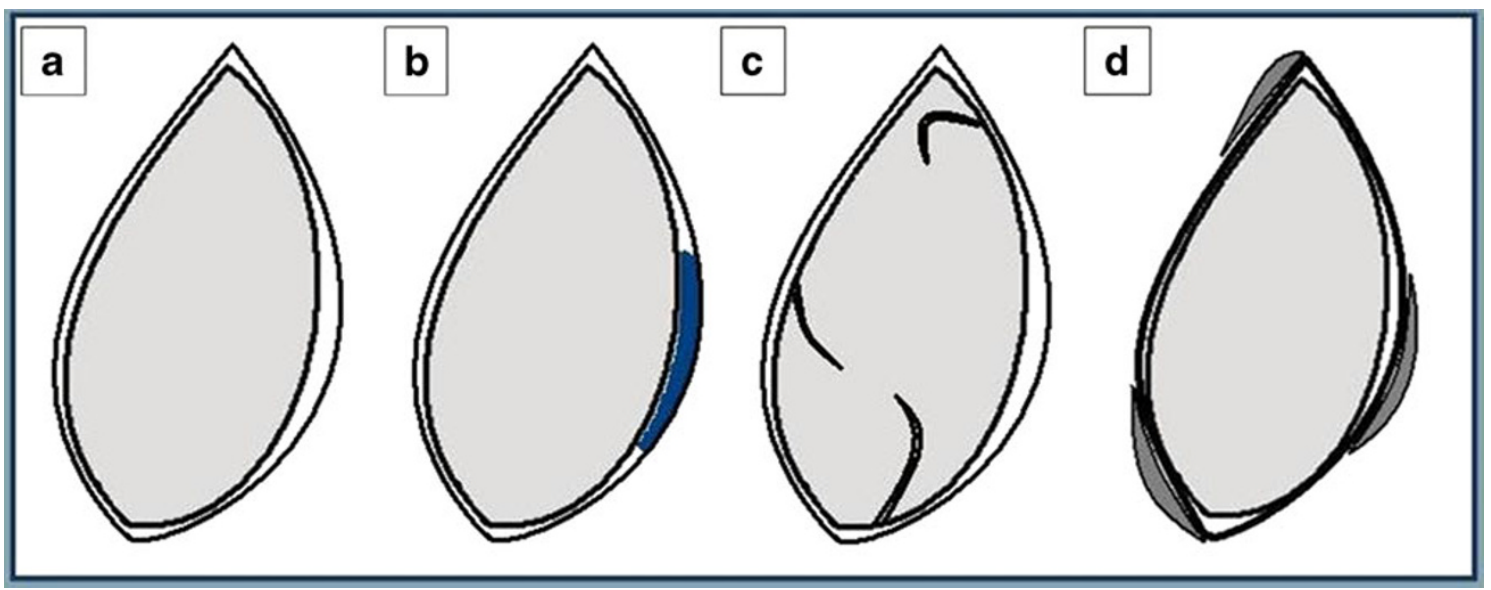

Fig. 13 Variants of normal breast implants. a Intact implant has an uninterrupted shell and fibrous capsule adjacent to the breast parenchyma. b Periprosthetic fluid. Presence of a small-to-moderate amount of reactive fluid surrounding the implant. c Simple or complex radial folds. Lines extending from the surface of the implant and inwards in a rather perpendicular manner. d Calcification and thickening of the fibrous capsule 


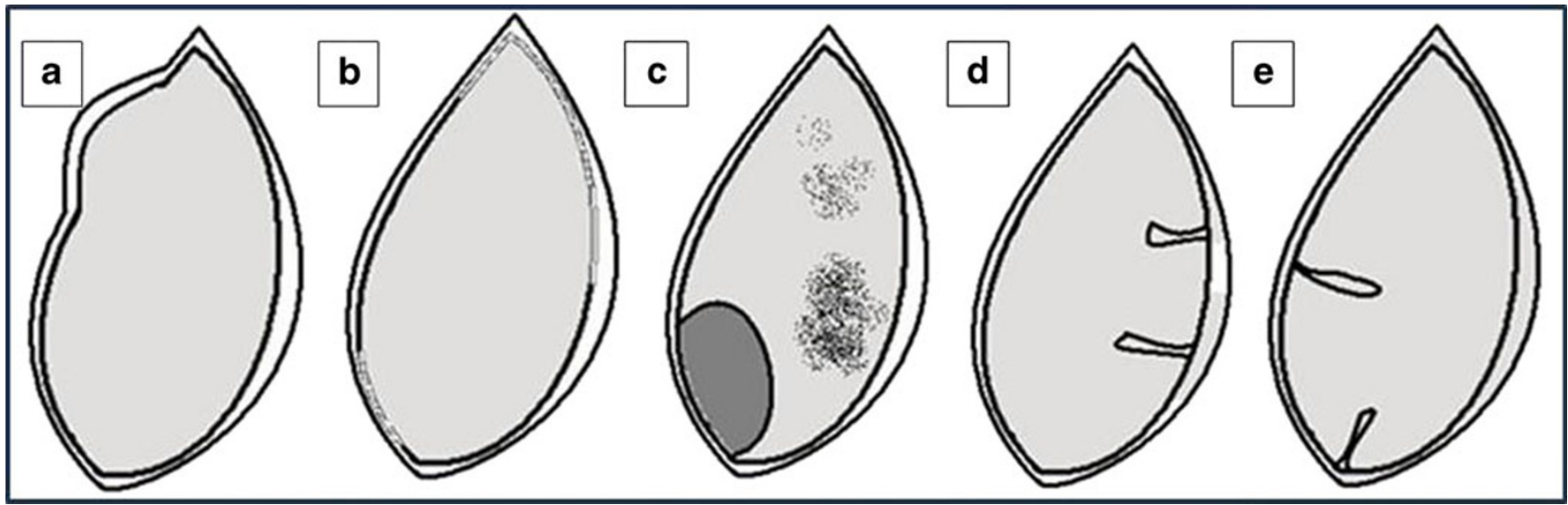

Fig. 14 Findings of possible breast implant rupture. a Deformity in contour. The border of the implant is bulging more than usual (called the "rat-tail sign" when very pronounced). Sometimes rupture cannot be differentiated from herniation. $\mathbf{b}$ Irregular margin. The border of the implant is blurry. Frequently seen with calcification of the fibrous capsule. c Changes in the signal intensity of the silicone gel. Water/

rupture, MRI shows free silicone outside the implant shell but still contained by the fibrous capsule. Focal silicone invagination between the inner shell and fibrous capsule are common, resulting in the "teardrop sign" and the "key-hole sign" $[5,7,9]$. An uncollapsed silicone implant rupture is defined as a tear of the silicone implant shell and is considered an intracapsular rupture (Fig. 7). Only these more subtle findings will be present in up to $52 \%$ of ruptured implants [15].

Extracapsular silicone implant rupture is defined as rupture of both the implant shell and the fibrous capsule, with macroscopic silicone leakage that extends beyond the fibrous capsule into surrounding tissues. Focal areas of high signal intensity in sequences that enhance only the silicone represent free silicone [5, 9, 12, 16] (Fig. 8). The linguine sign is often present with extracapsular ruptures.

Unlike rupture, gel bleed is microscopic silicone leakage through an intact implant shell [12]. This phenomenon is closely related to the chemical affinity between the outer shell of the silicone elastomer and the gel contained therein. Indeed, the gel, if in contact with the outer shell, can break the noncovalent molecular bonds between the polymer chains, causing swelling and weakening of the shell itself. Once it separates from its shell, silicone can migrate, even reaching the upper limbs, the liver, the inguinal lymph nodes, the synovium, the skin, and the pleura fluid. For this reason, the presence of silicone gel in regional lymph nodes can be due to gel bleed and not be always indicative of a present implant rupture. With the introduction of new cohesive gel implants, the phenomenon of gel bleeding has not been found [13]. Most normal transudation of microscopic amounts of silicone gel cannot be detected by MRI. Only when a gel bleed is extensive can silicone gel be detected serum mixed in the silicone gel through a defect in membrane. d "Noose sign" or "key-hole sign". Small invagination of the shell where the two membranes do not touch. e "Teardrop sign". Invagination of the shell containing a droplet of silicone. The last two images represent silicone gel leakage through a small focal implant shell tear

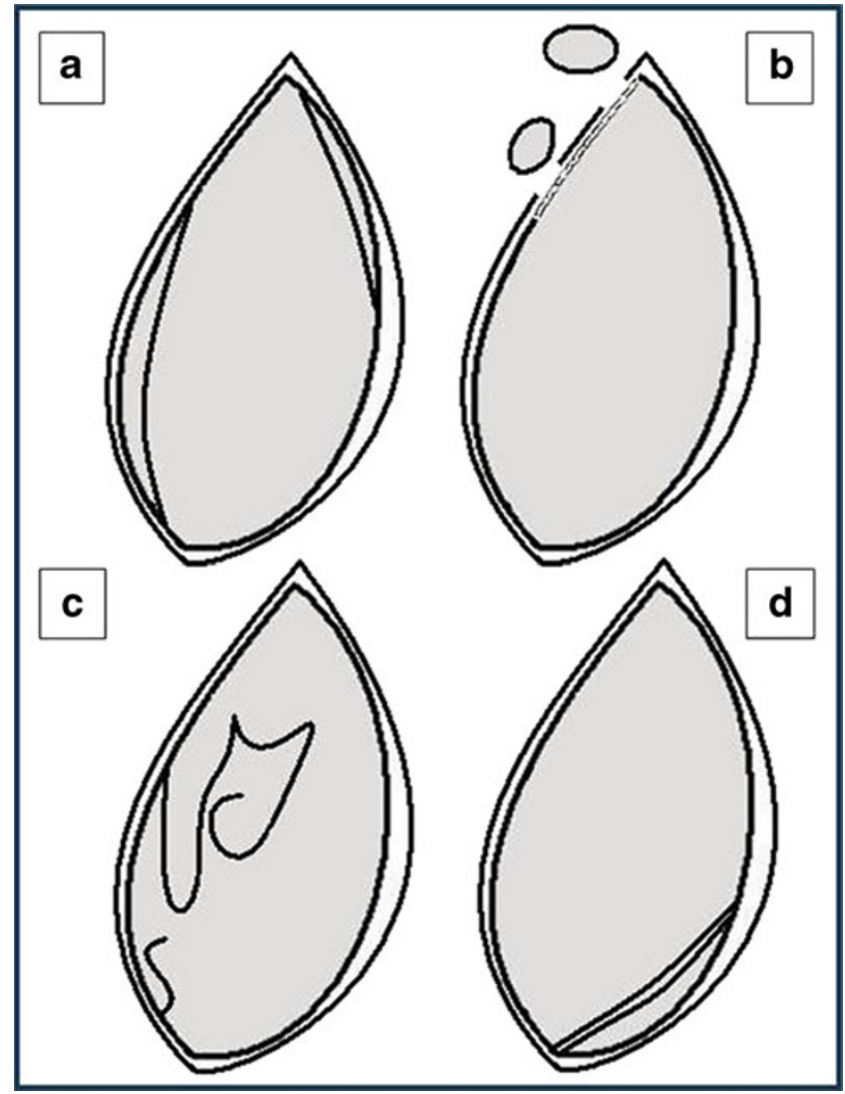

Fig. 15 Definitive findings of breast implant rupture. a Subcapsular lines. Lines running almost parallel to the fibrous capsule and just beneath it. The beginning and the end of the line can be followed to the surface of the implant. b Siliconomas and free silicone. Disruption of the shell and fibrous capsule will allow silicone to extravasate into surrounding breast tissue. c "Linguine sign". Folded wavy multidirectional lines within the silicone gel, representing the collapsed implant shell. d "Railroad track sign". Two parallel lines in close proximity forming a double-contoured subcapsular line within the silicone gel 
outside the implant shell and form the inverted teardrop sign. An uncollapsed implant rupture can have a similar or identical magnetic resonance appearance to a large gel bleed.

MRI often shows radial folds or normal infoldings of the shell extending to the periphery of the implant. These folds may appear prominent and complex, but should not be confused with implant rupture or leak. This is one of the major pitfalls and causes of false positives on MRI, mimicking the total collapse of the implant shell [9, 10, 13, 16]. Radial folds and periprosthetic fluid are considered normal variants of breast implants (Fig. 9). This reactive fluid probably results from an inflammatory response and is not indicative of implant rupture.

Radial folds are frequently observed in patients with capsular contracture. Capsular contracture is the most common complication. It is caused by excessive scar tissue around the implant that tightens and squeezes the implant. Occasionally, the capsule tears, allowing part of the implant to herniate into adjacent parenchyma. The capsule sometimes calcifies, and rigid calcium deposits may be palpated immediately adjacent to the implant (Fig. 10) [18].

Rarely, intracapsular rupture will show multiple hyperintense foci on T2-weighted images or multiple hypointense foci on water-suppression images within the implant lumen; these findings are referred to as the "salad oil sign" or "droplet sign". Without other MRI evidence of implant rupture, like the identification of the collapsed implant shell or free silicone within the breast parenchyma (with no previous history of implant rupture), water droplets or small amounts of air within a silicone implant are not reliable signs of implant rupture [9, 10, 16]. However, this sign should prompt the search for subtle signs of intracapsular rupture [16] (Figs. 11 and 12). The entire spectrum of MRI findings for silicone breast implants is illustrated in Figs. 13, 14 and 15.

Although MRI is the most accurate technique for evaluating implant integrity, it is important to know the mammographic, US and CT findings for implant rupture.

\section{Mammography}

Mammography is of little value in the assessment of implant integrity, although it may be useful for the assessment of the surrounding breast tissue [10]. Silicone implants should be evaluated on a yearly basis to detect any change in configuration or identify any new free silicone within the breast parenchyma. Mammographic features that
Fig. 16 Oblique mammograms in a 29-year-old transsexual with subglandular implants and silicone injections. Diffuse areas of increased density are visualised adjacent to the implant (arrows)
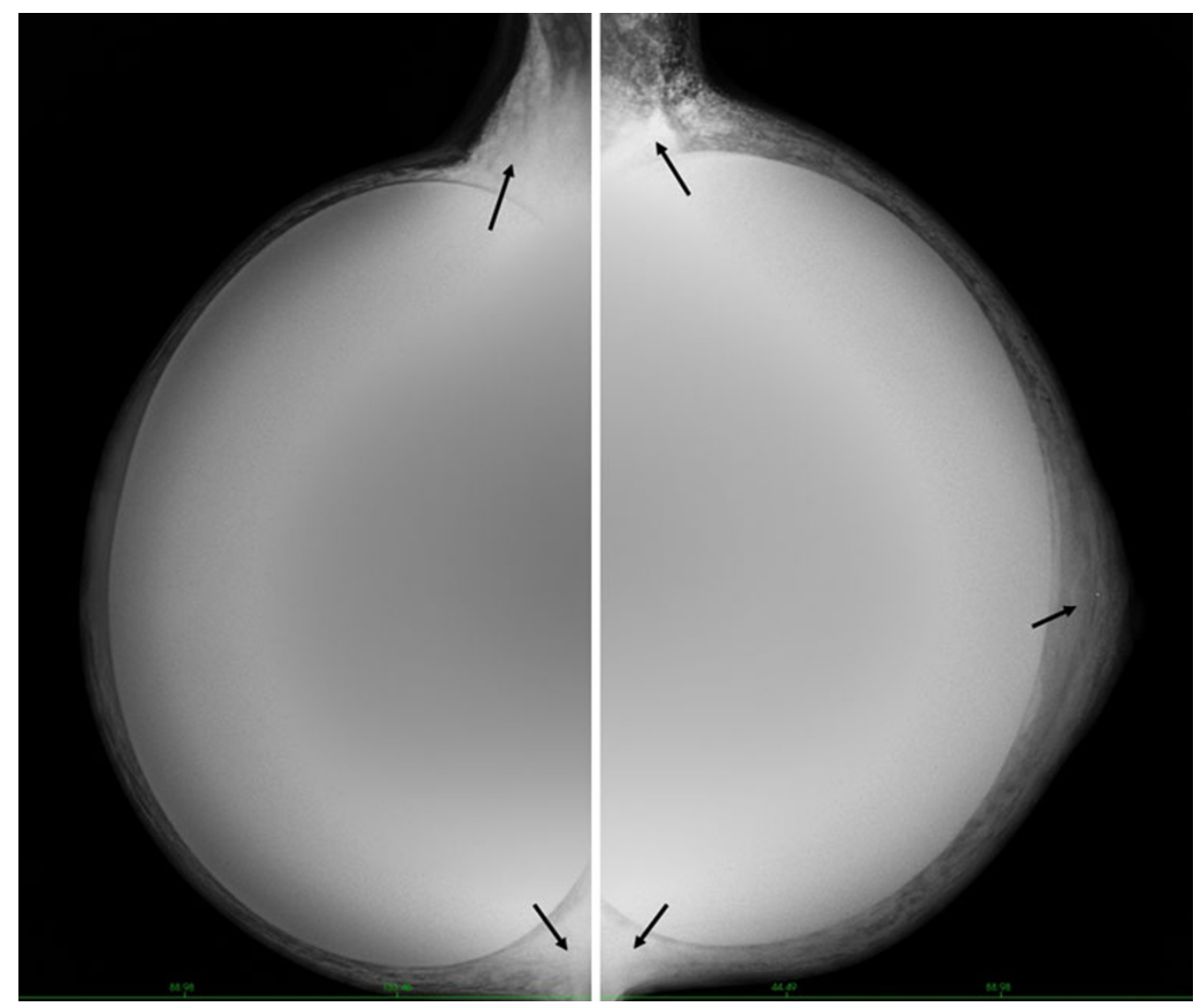
are not specific to implant rupture include a measurable periprosthetic dense band, periprosthetic calcification, asymmetry of implant size or shape, and focal herniation of the implant. Periprosthetic hyperdensity (Fig. 16) is significantly associated with extracapsular rupture $(p \leq 0.05)$ and inhomogeneous, reduced radiopacity seems to indicate rupture, without discriminating the type of rupture $(p \leq 0.05)$ [16]. Specific mammographic evidence of implant rupture is extravasation of silicone outside the implant shell [9].

Because mammography can easily detect free silicone within the breast parenchyma, extracapsular silicone implant ruptures can be identified. Unfortunately, silicone is dense and is not easily penetrated by the X-ray energies used for mammography; therefore, intracapsular rupture cannot be identified by mammography. The sensitivity of mammography for the rupture of silicone implants is variable but always low, ranging between $25-30 \%$ [10, $12]$ and $68 \%$ [14].

Several cases of silicone implant rupture from compression during a mammogram have been reported. Most probably occurred in women who had intracapsular ruptures previous to their mammogram [9].

The best mammograms are obtained when the breast is maximally compressed so the X-ray beam penetrates the thinnest possible layer of tissue. In 1988, Eklund et al. [13, 15] introduced the displacement technique to facilitate mammography in augmented women (Fig. 17). A thorough clinical history should be obtained before mammography to ensure that the patient has not had a previous implant rupture or has not had direct silicone injections.

\section{Ultrasonography}

Assessments of the usefulness of ultrasonography (US) for detecting implant ruptures vary widely. Although US does not use ionising radiation, it is operator dependent. Typically, US breast implant examination involves evaluation of morphology, contour and contents, periimplant tissues and axillae. Thus, the transversal-to-longitudinal ratio of the implants is calculated, the regularity of implant margins (radial folds) and the homogeneity of the implant lumen are checked, and signs of free silicone or granulomas in the breast or in the axillary lymph nodes are sought (Fig. 18) [17]. The most reliable sign of an intact implant is an anechoic interior [9]. A completely negative US examination strongly supports implant integrity, limiting the application of mammography or MRI to cases suspicious at US $[1,10]$.

Reverberation artefacts are commonly encountered in the anterior aspect of the implant and should not be confused

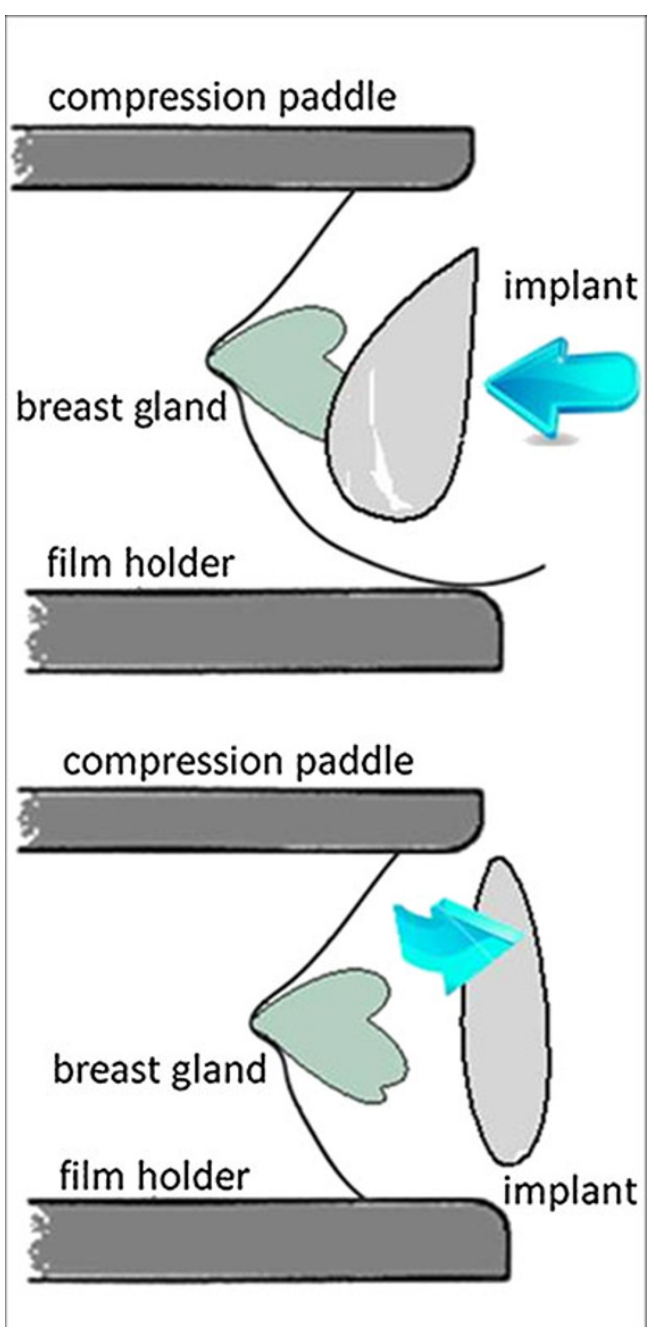

Fig. 17 Implant position is an important factor when studying the breasts. Patients with submammary implants have fewer visualised area compared to patients with submuscular implants. The displacement technique introduced by Eklund facilitates mammography in women with implants. Slightly more tissue is visualised with displacement (below) than with standard compression mammography (above)

with abnormalities. One limitation of US is that marked attenuation of the ultrasound beam by silicone hinders the evaluation of the back wall of an implant and the tissue posterior to it. Similarly, residual silicone granulomas from extracapsular rupture or from previous direct silicone injections compromise the evaluation of a new implant (Figs. 19 and 20).

Radial folds present as echogenic lines that extend from the periphery to the interior of the implant. These folds are normal infoldings of the implant membrane into the silicone gel. Small amounts of free silicone mixed within the surrounding breast tissues give rise to the characteristic echogenic "snowstorm" (statistically significant for extrac- 

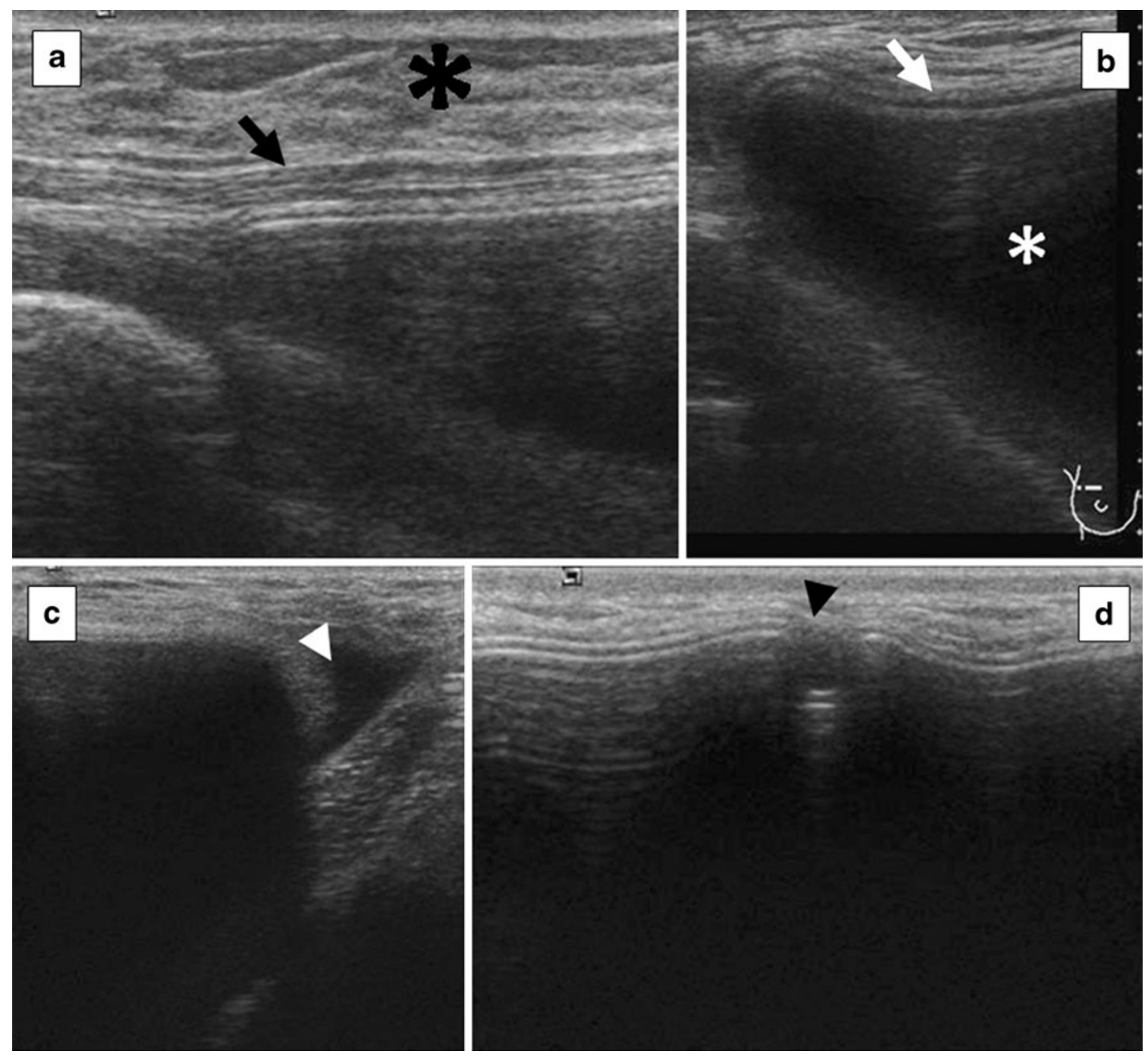

Fig. 18 a and b Ultrasonography of a woman with an intact implant. Breast gland (black asterisk), pectoralis major muscle (black arrow), and implant shell (white arrow) visualised as a thin and continuous echogenic line at the parenchymal tissue-implant interface, and silicone implant (white asterisk). (c) A small fluid collection around the implant (arrowhead) and (d) a simple infolding of the shell silicone implant (arrowhead)

significant for intracapsular rupture, $\mathrm{p} \leq 0.05$ [16]). This sign is analogous to the linguine sign seen on MRI, and it is the most reliable sign of intracapsular rupture [12]. It is important not to confuse the stepladder sign with normal prominent radial folds.

\section{Computed tomography}

At CT, an intact silicone implant is characterised by an oval shape and homogeneous grey density within a surrounding high-density ring. The implant often has contour deformities or implant bulges or hernias. The $\mathrm{CT}$ findings of intracapsular silicone implant rupture are similar to the MRI findings, and the linguine sign can also be seen at 
Fig. 19 (Above) A coronal maximum intensity projection from a silicone-excited sequence in a transsexual (Fig. 16) demonstrating multiple nodules with high signal throughout both breasts representing free silicone (arrows). (Below) It is extremely difficult to evaluate the silicone implants by ultrasonography because of attenuation of the ultrasound beam by the free injected silicone and granuloma formation in the subcutaneous tissue
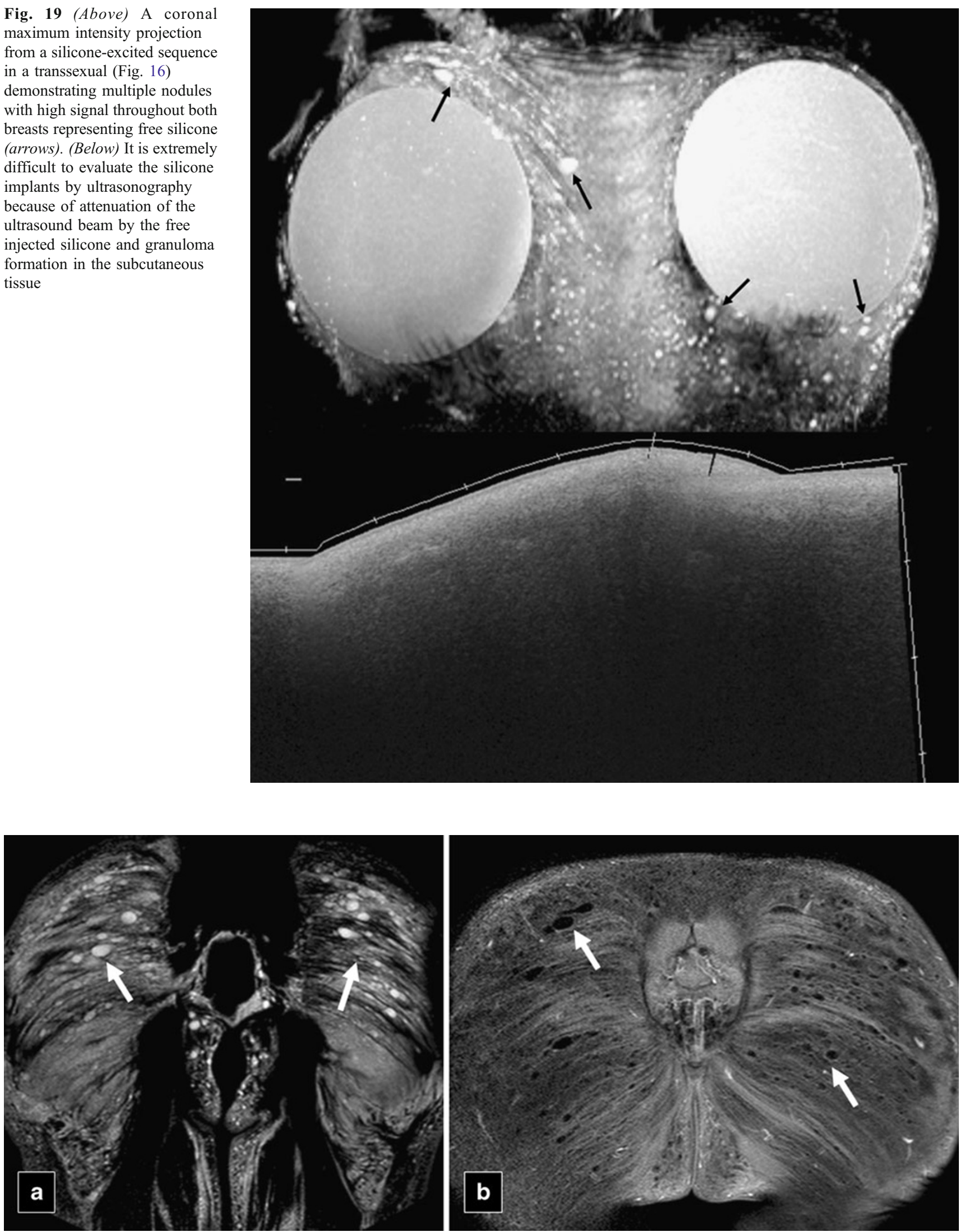

Fig. 20 a Coronal silicone-excited sequence and (b) coronal contrast-enhanced fat-suppressed T1-weighted image of the previous patient show multiple nodules of free silicone ("siliconomas") in the gluteal muscles 
Fig. 21 Extracapsular silicone implant rupture in a 52-year-old woman with a history of breast cancer who presented with a palpable lesion in the supraclavicular right region. Mammogram shows an irregular lump from the implant (arrowhead) and ultrasonography demonstrates the presence of a nodular lesion with typical inhomogeneity (the "snowstorm sign") at the posterior margin, suspicious for a lymph node containing silicone
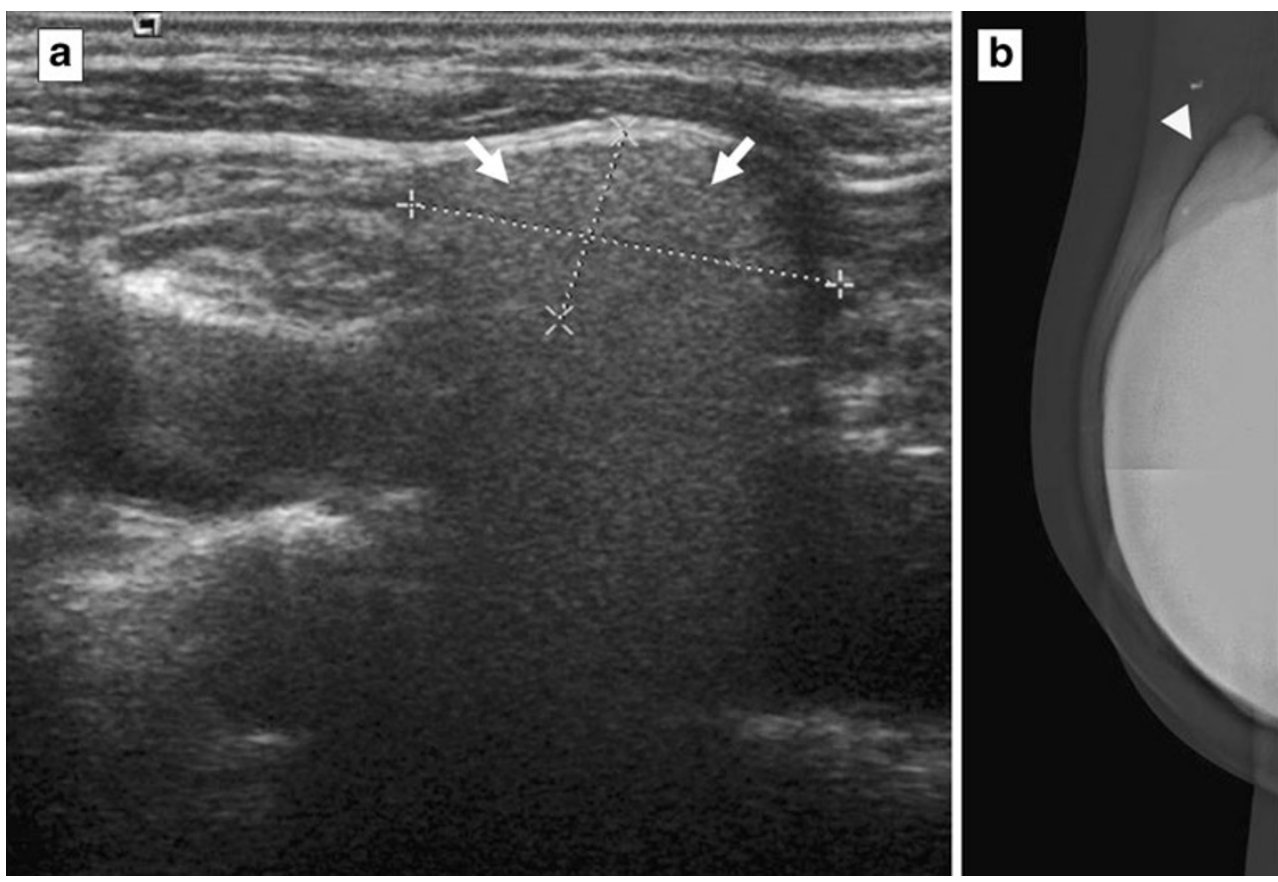

CT [8]. The collapsed implant shell can usually be easily identified (Figs. 23 and 24). Because silicone and soft tissues have similar radiodensities, extracapsular silicone can be difficult to identify on CT. However, in most cases of extracapsular silicone implant ruptures, CT can detect the collapsed implant shell, so the ruptured implant is not usually missed.

Nevertheless, CT uses ionising radiation; for this reason and its low sensitivity and specificity, CT should not be the study of choice, especially in young women. Most CT images of ruptured breast implants are incidental findings in asymptomatic patients or in already known failed implants.

\section{Additional lesions found in breast implant study}

All breast diseases can also occur in women with implants; among these, the most important for its prognosis and treatment is breast cancer. The risk of breast cancer is not increased in augmented women [11, 16], and there is no significant difference in breast cancer

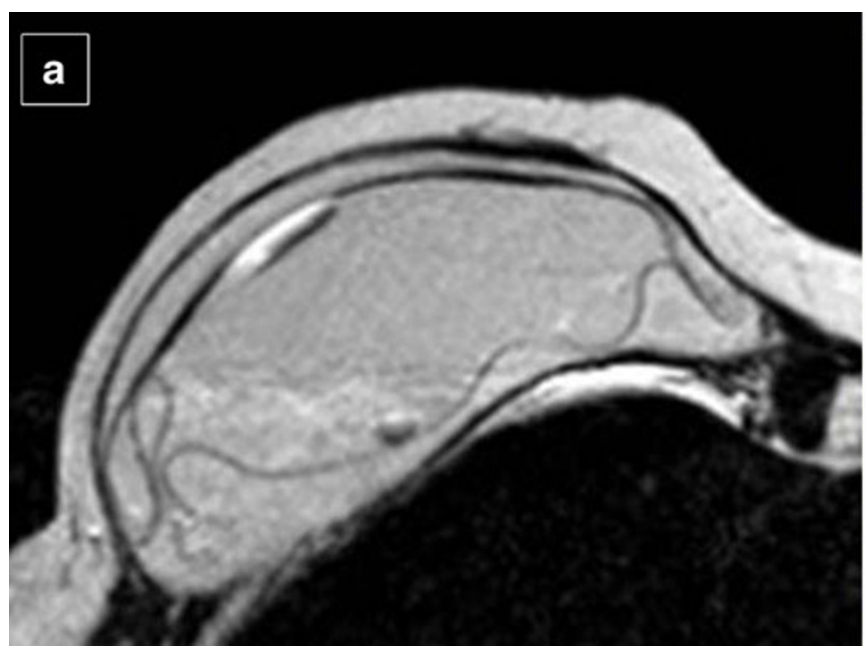

Fig. 22 Cytology of the node shows multinucleated foreign-body giant cells (arrowhead) with abundant birefringent particles inside and outside the cytoplasm, compatible with gel silicone (arrows). Axial

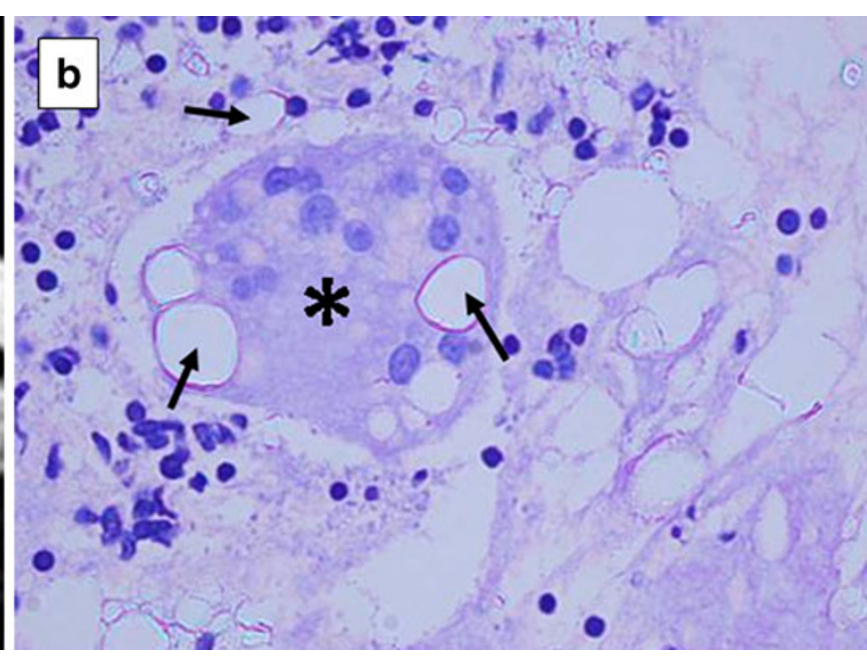

T2-weighted turbo spin-echo MRI study corroborated an extracapsular rupture of the implant 

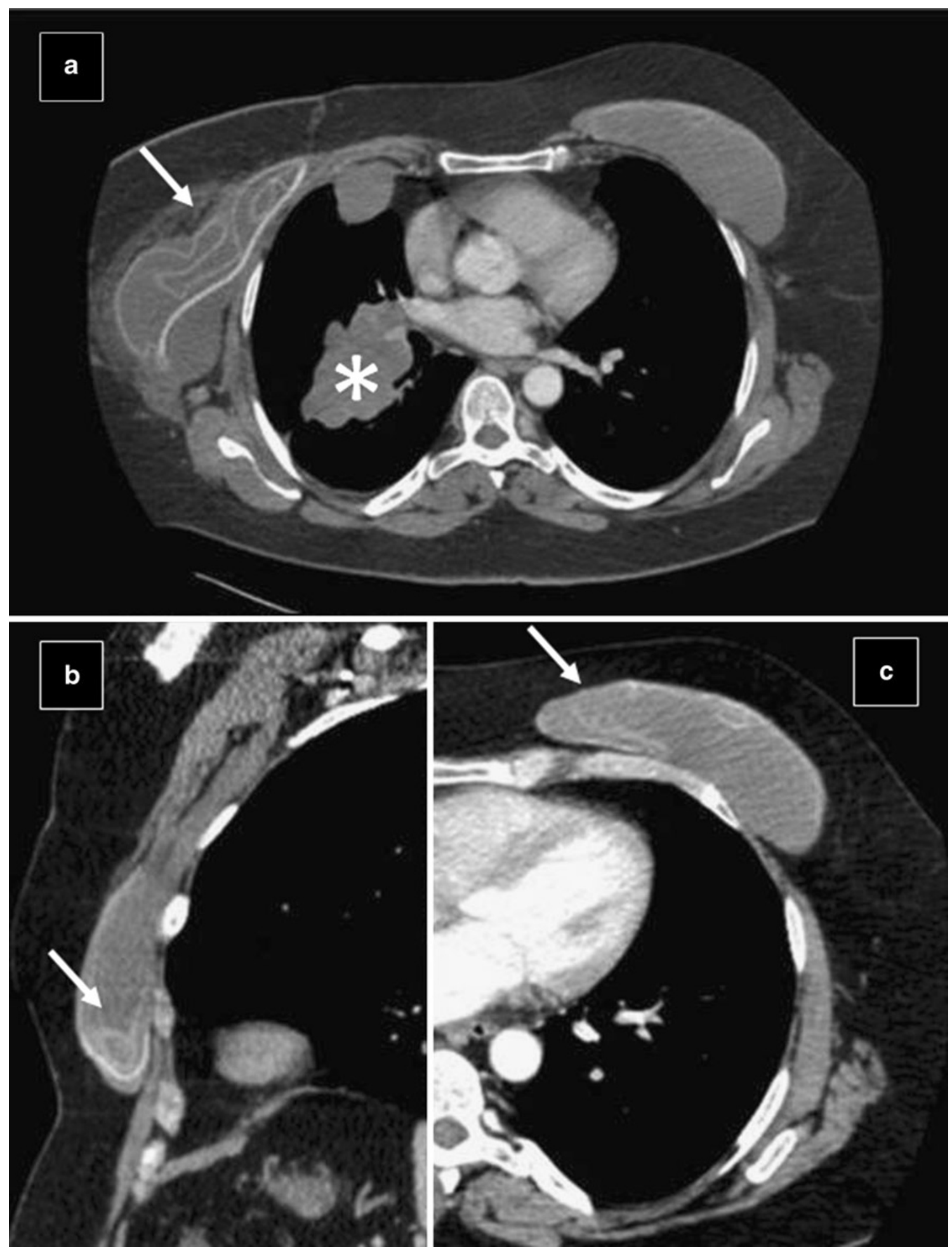

Fig. 23 Bilateral ruptured implants in a woman with primary lung cancer (asterisk). a Axial CT scan shows a severe deformity of the right implant surface representing a collapsed ruptured prosthesis

survival rates between women with and those without implants [11, 12]. However, because augmented women (arrow). b Sagittal multiplanar reconstruction and (c) axial CT scan of the left implant show high-density curvilinear lines within the implant ("linguine sign", arrows)

will sometimes develop breast cancer, there should be persistent precautions concerning cancer detection. Implants 
Fig. 24 Unilateral implant rupture. (a) Axial CT scan shows small high-density lines within the silicone gel in the right implant, suggestive of collapsed rupture (arrow). (b) Axial silicone-excited MRI sequence confirmed intracapsular rupture, showing hypointense wavy lines at the posterior margin of the right implant ("linguine sign") and subcapsular line at the anterior margin (arrows). Normal infoldings in the left implant (arrowhead)
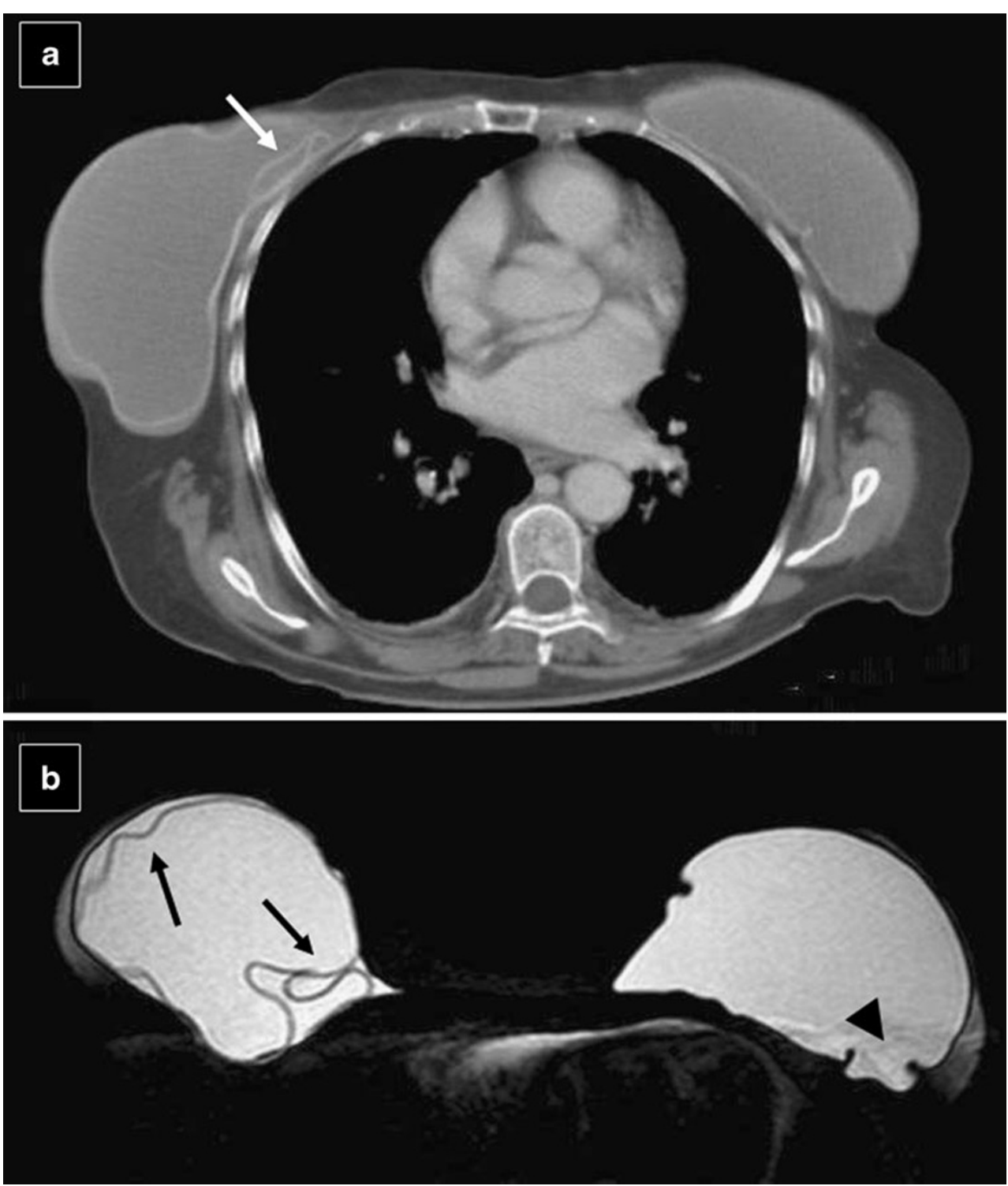

might interfere with the earliest possible detection of a cancer by altering physical examination of the breast or impairing various imaging techniques [18] (Figs. 25 and 26). The sensitivity of mammography and US for cancer detection may be reduced in augmented woman [1]. However, the presence of the implant does not seem to decrease the sensitivity of breast MRI [11, 14]. When evaluating these patients, physical and mammographic findings should be correlated. Any palpable abnormalities should be studied with ultrasound or contrast-enhanced MRI.

MRI is clearly recommended in augmented woman with suspicion of a tumor [1]. All suspicious lesions should be analysed by fine-needle aspiration or core needle biopsy with maximum caution to avoid damaging the implant (Fig. 27).

\section{Conclusion}

The risk of implant rupture increases with implant age. Radiologists need to be able to recognise the normal appearance of commonly used implants on various imaging techniques. The role of MRI in the evaluation of breast implants resides in implant integrity evaluation and breast cancer detection (especially in women with oncoplastic reconstructive surgery). Conventional breast imaging techniques, especially ultrasonography, still provide useful information about implants.

Intracapsular rupture, which is frequent but often clinically silent, cannot be easily detected by mammography because of the radiopacity of the implant. The sensitivity of ultrasonography is greater if discontinuous echogenic lines within silicone gel ("stepladder sign") are 
Fig. 25 Breast ultrasonography of a 39-year-old (a) and a 30-year-old (b) augmented women. In both studies there are two lesions (arrows) considered BIRADS III under follow-up. c Ultrasonography of a 46-yearold augmented woman with a history of breast cancer. US shows a lesion suspicious of recurrent tumor (arrow) that was confirmed by histology. Breast implant (asterisk)
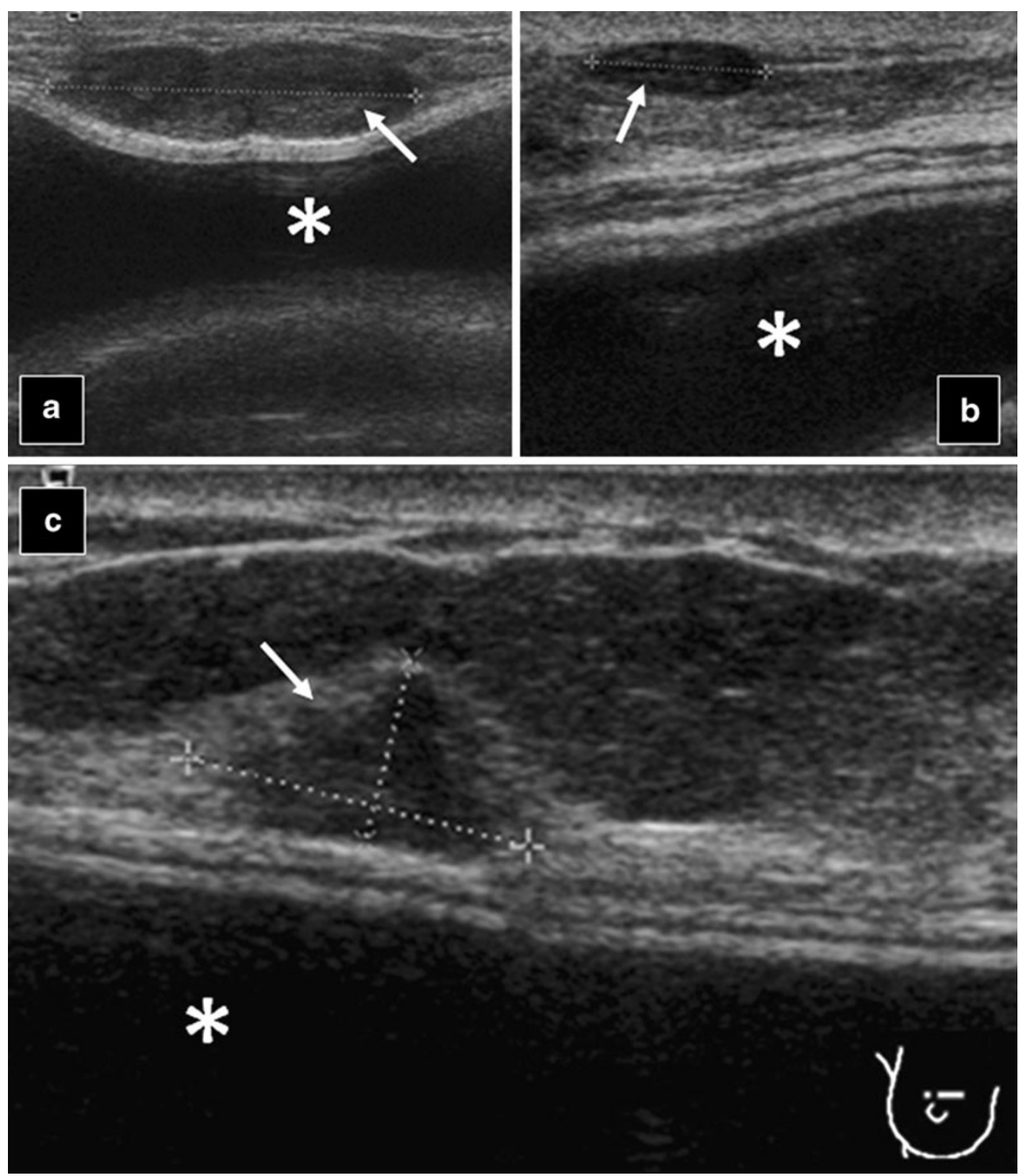

detected. Periprosthetic hyperdensity on mammography and the "snowstorm sign" on ultrasonography are significantly associated with extracapsular rupture.

MRI is more accurate than ultrasonography and mammography for assessing implant integrity. MRI is also the most accurate technique for differentiating intracapsular from extracapsular rupture and for assessing the extent of silicone leakage into the breast parenchyma and granuloma formation. MRI can reliably reveal collapsed intracapsular rupture by detecting shell fragments floating inside the silicone gel implant ("linguine sign"). This sign is also frequently associated with extracapsular rupture. An uncollapsed silicone implant rupture is seen as a sometimes subtle silicone invagination between the inner shell and fibrous capsule, known as the "teardrop sign" or the "keyhole sign". Other imaging signs, such as the "salad oil sign" or "droplet sign", are nonspecific and not reliable without other MRI evidence of implant rupture. However, these signs should prompt a search for subtle signs of intracapsular rupture. It is essential to be aware of the spectrum of normal appearances to avoid false-positive findings (prominent radial folds).

Moreover, the information provided by plastic surgeons is critical and often essential in the evaluation of breast implant integrity. The surgical technique of implantation, intraoperative complications that may have occurred, and the clinical monitoring of patients are very useful tools that the radiologist need to know about.

In general, in symptomatic patients, after conventional imaging, non-contrast MRI is recommended to confirm or exclude rupture. MRI is not recommended for screening for implant rupture in asymptomatic patients. In patients with oncoplastic surgery, dynamic contrast-enhanced MRI is indicated to evaluate the breast gland parenchyma. 
Fig. 26 An oblique mammogram of a 57 -year-old woman with a submammary implant (a). A cluster of suspicious microcalcifications can be identified in the breast gland (arrows). A magnified mammogram (b) confirms the presence of malignant microcalcifications (arrows)
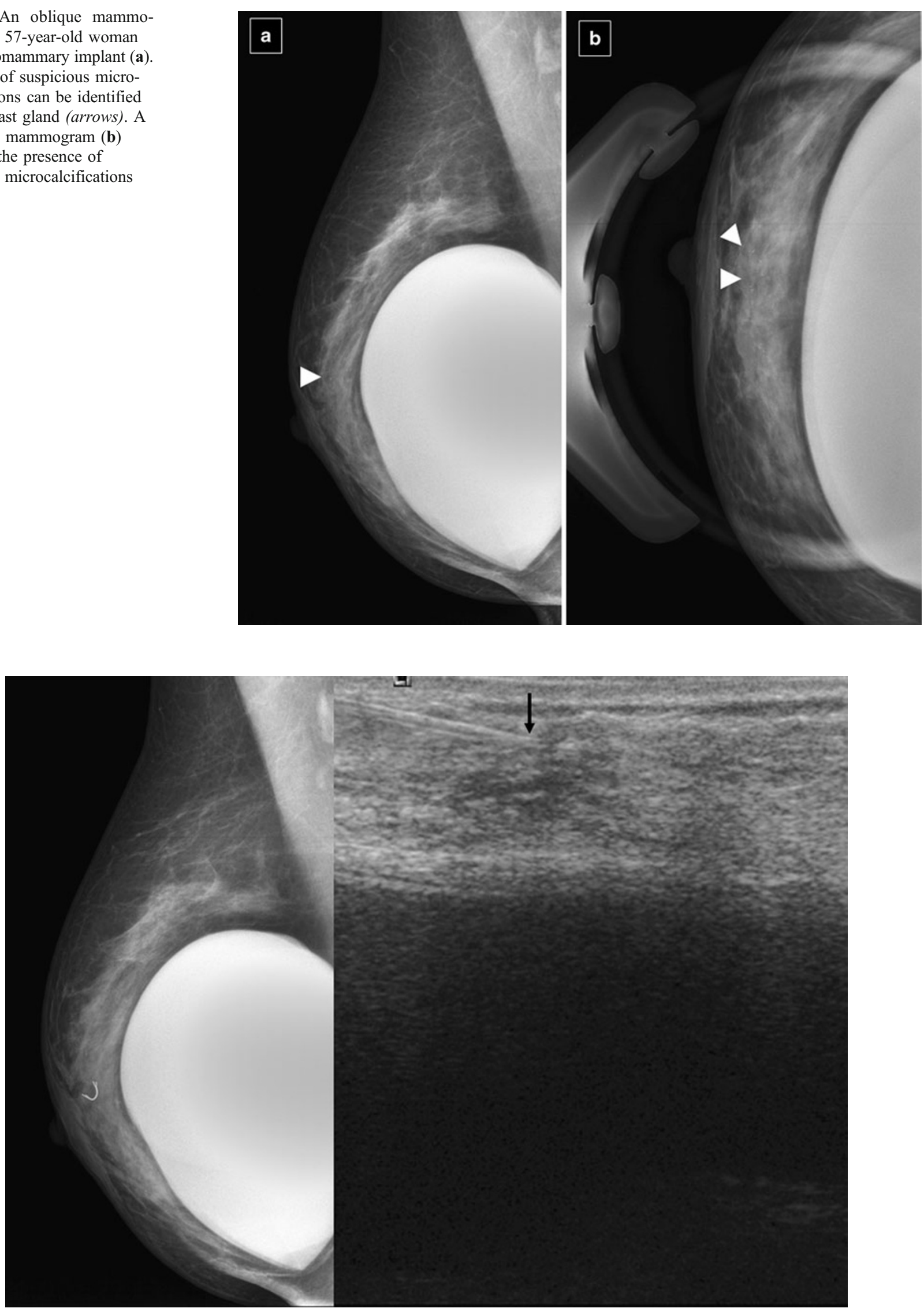

Fig. 27 (Left) Metallic clip placed in the microcalcifications site after biopsy. (Right) Real-time ultrasound is used to guide the needle tip (arrow) 
Acknowledgments The authors gratefully acknowledge the contribution of radiologists, technicians, oncologists and surgeons of the Hospital Dr Josep Trueta, without whose efforts this work would not have been possible.

The authors thank John Giba for the English correction and assistance.

\section{References}

1. Frank S, Mahdi R, Sherko K (2010) Imaging in patients with breast implants - results of the First International Breast (Implant) Conference 2009. Insights Imaging 1:93-97

2. Yasuo A, Ritsu A, Shinichiro K et al (2007) Silicone-selective multishot echo-planar imaging for rapid MRI survey of breast implants. Eur Radiol 17:1875-1878

3. Hölmich Lisbet R, Fryzek Jon P, Kim K et al (2005) The diagnosis of silicone breast-implant rupture clinical findings compared with findings at magnetic resonance imaging. Ann Plast Surg 54:583-589

4. Hölmich Lisbet R, Soren F, Fryzek Jon P et al (2003) Incidence of silicone breast implant rupture. Arch Surg 138:801-806

5. Hölmich Lisbet R, Vejborg Ilse M, Carsten C et al (2005) The diagnosis of breast implant rupture: MRI findings compared with findings at explantation. Eur J Radiol 53:213-225

6. Hölmich Lisbet R, Vejborg Ilse M, Carsten C et al (2004) Untreated silicone breast implant rupture. Plast Reconstr Surg 114:204-214

7. Herborn Cristoph U, Borut M, Daniel E et al (2002) Breast augmentation and reconstructive surgery: MR imaging of implant rupture and malignancy. Eur Radiol 12:2198-2206
8. Chul TK, Sun JH, Suk RT et al (2005) Analysis of 30 breast implant rupture cases. Aesth Plast Surg 29:460-469

9. Gorczyca David P, Gorczyca Stephanie M, Gorczyca Kathryn (2007) The diagnosis of silicone breast implant rupture. Plast Reconstr Surg 120(suppl1):49S-61S

10. Glynn C, Litherland J (2008) Imaging breast augmentation and reconstruction. Br J Radiol 81:587-595

11. Sardanelli $\mathrm{F}$ et al. (2010) Magnetic resonance imaging of the breast: recommendations from the EUSOMA working group. Eur J Cancer. May;46(8):1296-316

12. Yang N, Muradali D (2011) The augmented breast: a pictorial review of the abnormal and unusual. AJR Am J Roentgenol. Apr;196(4):W451-460

13. Colombo G et al. (2011) Prosthetic Breast Implant Rupture: ImagingPictorial Essay. Aesth Plast Surg. Apr 13 [Epub ahead of print]

14. Mann RM, Kuhl CK, Kinkel K, Boetes C (2008) Breast MRI: guidelines from the European Society of Breast Imaging. Eur Radiol 18:1307-1318

15. Berg Wendie A, Khanh NT, Middleton MS et al (2002) MR imaging of extracapsular silicone from breast implants: diagnostic pitfalls. AJR Am J Roentgen 178:465-472

16. Giovanni Di Benedetto, Sara C, Luca G et al (2008) Comparative study of breast implant rupture using mammography, sonography, and magnetic resonance imaging: correlation with surgical findings. Breast J 14:532-537

17. Anna C, Carolina M, Chiara I et al (2006) Ultrasonographic appearance of breast implant complications. Ann Plast Surg $56: 243-247$

18. Handel Neal (2007) The effect of silicone implants on the diagnosis, prognosis, and treatment of breast cancer. Plast Reconstr Surg 120(suppl1):81S-93S 